Provided for non-commercial research and education use. Not for reproduction, distribution or commercial use.

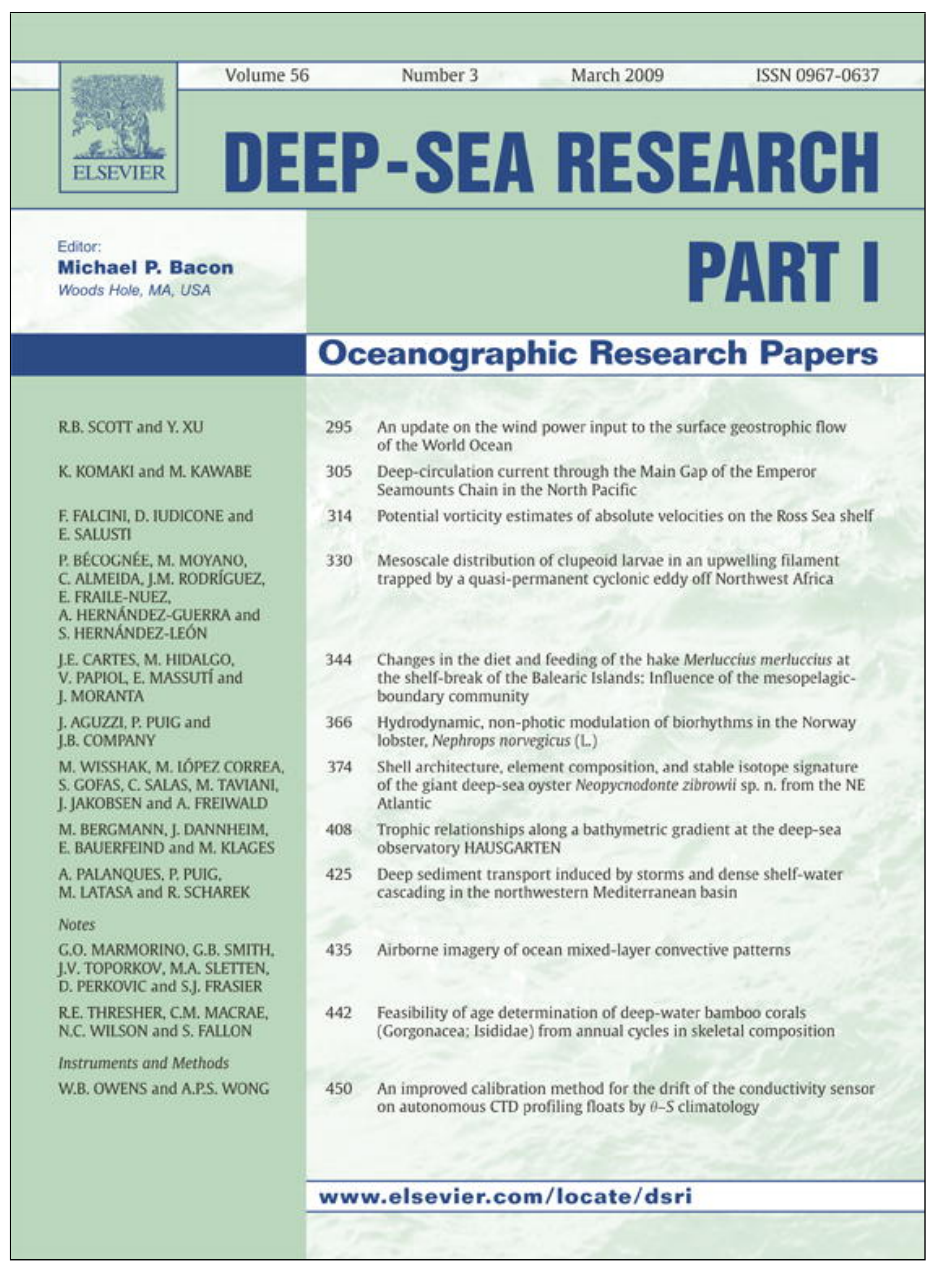

This article appeared in a journal published by Elsevier. The attached copy is furnished to the author for internal non-commercial research and education use, including for instruction at the authors institution and sharing with colleagues.

Other uses, including reproduction and distribution, or selling or licensing copies, or posting to personal, institutional or third party websites are prohibited.

In most cases authors are permitted to post their version of the article (e.g. in Word or Tex form) to their personal website or institutional repository. Authors requiring further information regarding Elsevier's archiving and manuscript policies are encouraged to visit:

http://www.elsevier.com/copyright 


\title{
Trophic relationships along a bathymetric gradient at the deep-sea observatory HAUSGARTEN
}

\author{
Melanie Bergmann *, Jennifer Dannheim ${ }^{1}$, Eduard Bauerfeind, Michael Klages \\ Alfred Wegener Institute for Polar and Marine Research, Am Handelshafen 12, D-27570 Bremerhaven, Germany
}

\section{A R T I C L E I N F O}

\section{Article history:}

Received 16 April 2008

Received in revised form

16 October 2008

Accepted 21 October 2008

Available online 5 November 2008

\section{Keywords:}

Arctic

Deep sea

Trophic level

Food web

Megafauna

Nitrogen stable isotope

Deposit feeder

Suspension feeder

\begin{abstract}
A B S T R A C T
Deep-seafloor communities, especially those from the ice-covered Arctic, are subject to severe food limitation as the amount of particulate organic matter (POM) from the surface is attenuated with increasing depth. Here, we use naturally occurring stable isotope tracers $\left(\delta^{15} \mathrm{~N}\right)$ to broaden our rudimentary knowledge of food web structure and the response of benthic organisms to decreasing food supplies along the bathymetric transect ( $1300-5600 \mathrm{~m}$ water depth) of the deep-sea observatory HAUSGARTEN. Encompassing five trophic levels, the HAUSGARTEN food web is among the longest indicating continuous recycling of organic material typical of food-limited deep-sea ecosystems. The $\delta^{15} \mathrm{~N}$ signatures ranged from 3.0\%o for Foraminifera to $21.4 \%$ ( \pm 0.4$)$ for starfish (Poraniomorpha tumida). The majority of organisms occupied the second and third trophic level. Demersal fish fed at the third trophic level, consistent with results from stomach contents analysis. There were significant differences in the $\delta^{15} \mathrm{~N}$ signatures of different functional groups with highest $\delta^{15} \mathrm{~N}$ values in predators/ scavengers $(13.2 \pm 0.2 \%$ ) followed by suspension feeders $(11.2 \pm 0.2 \%$ ) and deposit feeders $(10.2 \pm 0.3 \%$ ). Depth ( $=$ increasing food limitation) affected functional groups in different ways. While the isotopic signatures of predators/scavengers did not change, those of suspension feeders increased with depth, and the reverse was found for deposit feeders. In contrast to the results of other studies, the $\delta^{15} \mathrm{~N}$ signatures in POM samples obtained below $800 \mathrm{~m}$ did not vary significantly with depth indicating that changes in $\delta^{15} \mathrm{~N}$ values are unlikely to be responsible for the depth-related $\delta^{15} \mathrm{~N}$ signature changes observed for benthic consumers. However, the $\delta^{15} \mathrm{~N}$ signatures of sediments decreased with increasing depth, which also explains the decrease found for deposit feeders. Suspension feeders may rely increasingly on particles trickling down the HAUSGARTEN slope and carrying higher $\delta^{15} \mathrm{~N}$ signatures than the decreasing POM supplies, which elevates the $\delta^{15} \mathrm{~N}$ value of their tissues. Our results imply that a depth-stratified approach should be taken to avoid a misinterpretation of data obtained at different depths.
\end{abstract}

(c) 2008 Elsevier Ltd. All rights reserved.

\footnotetext{
* Corresponding author. Tel.: +4947148311739; fax: +4947148311776 .

E-mail address: Melanie.Bergmann@awi.de (M. Bergmann).

${ }^{1}$ Present address: Institute of Marine Research, PO Box 6404, 9294 Tromsø, Norway.
}

\section{Introduction}

Most deep-sea ecosystems rely on the vertical particle flux from the euphotic zone to the seafloor, and on particles that are transported along the slope (Klages et al., 2003). Large food particles ('food falls') such as mammal carcasses, fish, and large invertebrates may also play a significant role in the vertical carbon flux to the seafloor (Smith, 1985; Jones et al., 1998; Klages et al., 
2001; Soltwedel et al., 2003). Although the deep seafloor is considered the world's largest sink for biogenic carbon, only $1-10 \%$ of the material produced at the surface reaches the bottom (Klages et al., 2003; De La Rocha and Passow, 2007), as particles are exposed to biodegradation during sedimentation. Biodegradation also affects the biochemical properties of POM (particulate organic matter) reaching the sea bed (Rau et al., 1990; Mintenbeck et al., 2007). Deep-seafloor communities are thus subject to severe energy limitation (Gage and Tyler, 1991). It is still poorly understood how the deep-sea community is sustained despite the restricted energy input from the water column (Smith, 1987; Bailey et al., 2007). In general, most deep-sea organisms are deposit feeders (Jumars and Wheatcroft, 1989; Iken et al., 2001). Although our knowledge about the feeding ecology of benthic deep-sea species has expanded, few studies have explored the overall structure of deep-sea food webs (Iken et al., 2001; Polunin et al., 2001; Sahling et al., 2003).

Still less is known about deep-sea environments from polar regions which are frequently covered by ice and are thus even less accessible (Iken et al., 2005; Mintenbeck et al., 2007). Along with phytoplankton, which is the main source of production in open oceanic waters, ice algae also contribute to the net primary production in the Arctic (McMahon et al., 2006; Søreide et al., 2006; Tamelander et al., 2006), but we currently do not know what quantities reach the deep seafloor and to what extent they subsidise the fauna. Productivity is low because the annual sunlight cycle and sea ice limit the light available to primary producers. In addition, the deep Arctic is-like other regions of the ocean-characterised by a great retention of carbon in the twilight zone (Wassmann et al., 2003; Buesseler et al., 2007) further reducing food availability compared to open ocean waters (Klages et al., 2003; Sakshaug, 2003).

Climate change is the prominent global environmental threat of the 21st Century (IPCC, 2007) and will have severe impacts on the carbon flux to the deep seafloor (Smith et al., 2008), especially in the Arctic (Grebmeier et al., 2006). However, current predictions carry high levels of uncertainty as even crucial baseline information is scarce. It was in this context that the first and by now only long-term deep-sea observatory at high latitude, 'HAUSGARTEN', was established in the eastern Fram Strait (Soltwedel et al., 2005). Previous research along the bathymetric transect of HAUSGARTEN (1200-5600 m) has shown a decrease of organic carbon content, phytodetrital matter (Soltwedel et al., 2005) and bacterial abundance and viability (Quéric et al., 2004) with increasing water depth. Likewise, there was a stepwise decrease in meiofaunal densities and a decrease in macroand megafaunal densities at nearby HAUSGARTEN stations as a function of depth (Jaeckisch, 2004; WłodarskaKowalczuk et al., 2004; Hoste et al., 2007).

The main objective of this study was to assess deep-sea food web structure along a bathymetric gradient at a high northern latitude location using the stable isotope approach. This enables us to assess how decreasing food availability affects food web structure, as the food supply at HAUSGARTEN decreases with increasing depth. In the long run, this may allow us to fathom how changes at one level may impinge on other compartments of the ecosystem.

\section{Material and methods}

\subsection{Study area}

All samples were taken at 'HAUSGARTEN', a deep-sea observatory at high latitude established in the eastern Fram Strait, west of Svalbard at $79^{\circ} \mathrm{N}$ in 1999 (Fig. 1). HAUSGARTEN consists of nine stations along a bathymetric gradient from the upper slope of the Svalbard Margin $(1200 \mathrm{~m})$ to the Molloy Deep $(5600 \mathrm{~m})$ and of a latitudinal transect of seven stations along the $2500 \mathrm{~m}$ isobath starting at the margin of the sea ice zone (Soltwedel et al., 2005). While the shallower stations $(1200-3000 \mathrm{~m})$ are located along a gentle slope, those beneath are found on a steep slope (Hoste et al., 2007). Samples taken along these transects annually since 2000 have been used to determine a variety of faunal and environmental parameters.

The HAUSGARTEN area is affected by warm Atlantic waters transported by the West Spitsbergen Current in the upper $500 \mathrm{~m}$, which is fed by the North Atlantic Current (Schauer et al., 2008) such that the area is ice-free most of the year. Part of the HAUSGARTEN region is covered by ice during winter, but ice can also be present during summer (Bauerfeind et al., in revision). Below the warm Atlantic water layer, there are low-temperature waters modified by polar water masses (Schlichtholz and Houssais, 2002).

\subsection{Stable isotope analysis}

The utility of the stable isotope approach lies in the fact that stable isotope ratios in the proteins of consumers reflect those of the proteins in their diet in a predictable manner. Conventionally expressed as $\delta^{15} \mathrm{~N}(\%)$, the ratio of the nitrogen isotopes ${ }^{15} \mathrm{~N}$ to ${ }^{14} \mathrm{~N}$ generally exhibits a stepwise enrichment between 2.5 and 5\% per trophic step (Bearhop et al., 2004). The ratio of ${ }^{13} \mathrm{C}$ to ${ }^{12} \mathrm{C}\left(\delta^{13} \mathrm{C}\right)$ indicates the distance to the primary carbon source. Among other things, it is affected by an organism's lipid content (Mintenbeck et al., 2008). Stable isotope ratios can thus be used to reconstruct food webs and determine the trophic level of consumers. As we did not extract lipids prior to analyses we focus on $\delta^{15} \mathrm{~N}$.

\subsection{Sampling procedure}

Most samples were taken by the research ice breaker Polarstern (Table 1). To assess the $\delta^{15} \mathrm{~N}$ of primary producers, we took water samples in August 2005 at HAUSGARTEN station IV (Table 1 ) by a CTD/rosette system from 10, 20, 30 and $50 \mathrm{~m}$ water depth. A volume of 0.5-2 1 seawater was filtered onto pre-combusted $\left(500{ }^{\circ} \mathrm{C} / 12 \mathrm{~h}\right.$ ) glass-fibre filters (Whatman GF/F, $\varnothing 25 \mathrm{~mm}$ ) which were stored at $-20^{\circ} \mathrm{C}$. Prior to analysis, the filters were dried $\left(60^{\circ} \mathrm{C} / 12 \mathrm{~h}\right)$, pelletised and stored in an exsiccator. 


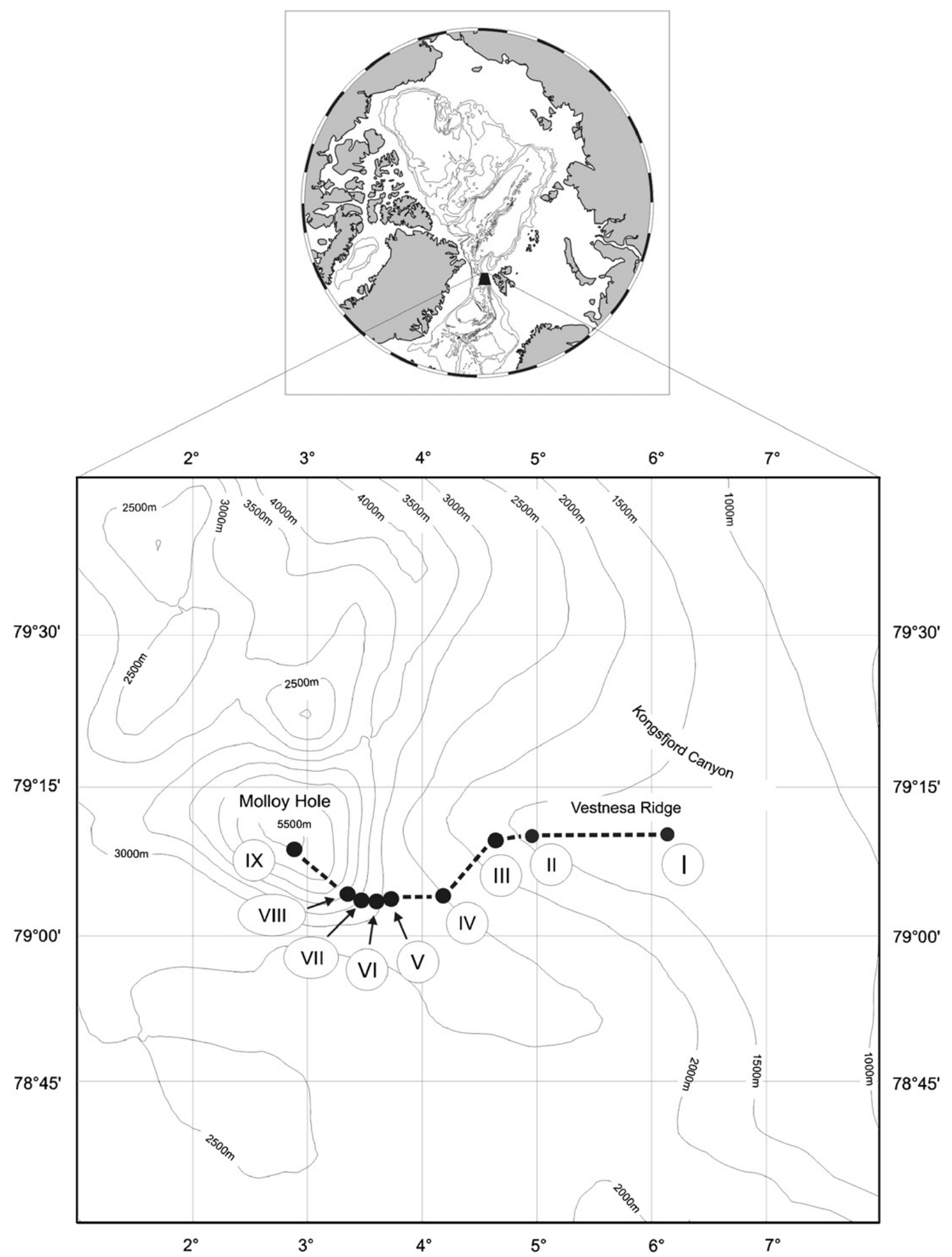

Fig. 1. Location of HAUSGARTEN and map of sampling stations HG I-IX distributed along the bathymetric transect (1200-5600 m).

Deep-sea fauna rely chiefly on particles sinking from the productive surface waters to the sea floor as their main source of carbon. To determine a $\delta^{15} \mathrm{~N}$ reference for benthic species, analyses of $\delta^{15} \mathrm{~N}$ were performed on POM collected by two year-round moored time-series sediment traps $(800 \mathrm{~m}$ water depth and $200 \mathrm{~m}$ above bottom) in 2002-2003 and a sediment trap attached to a benthic lander ( $2.5 \mathrm{~m}$ above bottom) at HAUSGARTEN IV in 2004/2005. The samples were poisoned in situ with mercury chloride $(0.14 \%)$. After retrieval of the traps, the samples were refrigerated and divided by a wet-splitting procedure with a rotating splitter after removal of the swimmers under a dissecting microscope (Bodungen et al., 1991). Sub-samples were then filtered 
Table 1

List of stations sampled for food-web analysis in chronological order. (Lat) latitude, (Lon) longitude.

\begin{tabular}{|c|c|c|c|c|c|c|c|}
\hline Expedition & Station No & Station & Position Lat (N) & Position Lon (E) & Depth (m) & Date (dd/mm/yy) & Sampling gear \\
\hline ARKXVIII/1b & PS62/179-2 & HG IV & $79^{\circ} 1.04^{\prime}$ & $4^{\circ} 19.77^{\prime}$ & 2593 & $07 / 08 / 02-22 / 07 / 03$ & Sediment trap mooring \\
\hline ARKXX/1a & PS66/103-1 & HG I & $79^{\circ} 8.97^{\prime}$ & $5^{\circ} 56.35^{\prime}$ & 1323 & $07 / 07 / 04$ & Agassiz trawl \\
\hline ARKXX/1a & PS66/118-1 & HG IV & $79^{\circ} 9.75^{\prime}$ & $3{ }^{\circ} 52.21^{\prime}$ & 2377 & 09/07/04 & Agassiz trawl \\
\hline ARKXXI/1b & PS68/240-1 & HG IV & $79^{\circ} 0.81^{\prime}$ & $4^{\circ} 20.51^{\prime}$ & 2590 & $12 / 07 / 04-19 / 08 / 05$ & Sediment trap mooring \\
\hline ARKXXI/1b & PS68/238-4 & HG IV & $79^{\circ} 4.55^{\prime}$ & $4^{\circ} 6.52^{\prime}$ & 2504 & $19 / 08 / 05$ & Bottom lander \\
\hline ARKXXI/1b & PS68/245-1 & HG IV & $79^{\circ} 3.79^{\prime}$ & $4^{\circ} 10.63^{\prime}$ & 2471 & $20 / 08 / 05$ & CTD/rosette \\
\hline ARKXXI/1b & PS68/247-1 & HG V & $79^{\circ} 5.67^{\prime}$ & $3^{\circ} 38.68^{\prime}$ & 3072 & $20 / 08 / 05$ & Agassiz trawl \\
\hline ARKXXI/1b & PS68/247-2 & HG V & $79^{\circ} 5.67^{\prime}$ & $3^{\circ} 38.68^{\prime}$ & 3072 & $20 / 08 / 05$ & Agassiz trawl \\
\hline ARKXXI/1b & PS68/256-1 & HG VII & $79^{\circ} 4.10^{\prime}$ & $3^{\circ} 29.41^{\prime}$ & 3870 & $22 / 08 / 05$ & Agassiz trawl \\
\hline ARKXXI/1b & PS68/260-1 & HG VI & $79^{\circ} 5.81^{\prime}$ & $3^{\circ} 33.27^{\prime}$ & 3576 & $22 / 08 / 05$ & Agassiz trawl \\
\hline ARKXXI/1b & PS68/270-1 & HG VI & $79^{\circ} 5.19^{\prime}$ & $3^{\circ} 34.82^{\prime}$ & 3430 & $24 / 08 / 05$ & Bottom lander \\
\hline ARKXXI/1b & PS68/272-2 & HG III & $79^{\circ} 5.31^{\prime}$ & $4^{\circ} 46.36^{\prime}$ & 1913 & $24 / 08 / 05$ & Agassiz trawl \\
\hline ARKXXI/1b & PS68/276-1 & HG II & $79^{\circ} 7.24^{\prime}$ & $4^{\circ} 58.30^{\prime}$ & 1546 & $25 / 08 / 05$ & Agassiz trawl \\
\hline ARKXXI/1b & PS68/278-1 & HG IV & $79^{\circ} 4.84^{\prime}$ & $4^{\circ} 25.05^{\prime}$ & 2293 & $26 / 08 / 05$ & Bottom lander \\
\hline AWI-ROV2005 & Victor-255 & HG IX & $79^{\circ} 06.08$ & $3^{\circ} 06.71^{\prime}$ & 5375 & $20 / 09 / 05$ & ROV slurp gun \\
\hline AWI-ROV2005 & Victor-256 & HG IV & $79^{\circ} 04.7^{\prime}$ & $4^{\circ} 08.1^{\prime}$ & 2467 & $24 / 09 / 05$ & ROV slurp gun \\
\hline ARKXXII/1C & PS70/ 200-1 & HGIV & $79^{\circ} 44.19^{\prime}$ & $4^{\circ} 25.66^{\prime}$ & 2644 & $17 / 07 / 07$ & Multiple corer \\
\hline ARKXXII/1c & PS70/159-1 & HGII & $79^{\circ} 7.82^{\prime}$ & $4^{\circ} 53.65^{\prime}$ & 1565 & $11 / 07 / 07$ & Multiple corer \\
\hline ARKXXII/1C & PS70/163-1 & HGI & $79^{\circ} 8.07^{\prime}$ & $5^{\circ} 59.45^{\prime}$ & 1304 & $12 / 07 / 07$ & Multiple corer \\
\hline ARKXXII/1c & PS70/169-1 & HGIV & $79^{\circ} 4.42^{\prime}$ & $4^{\circ} 14.35^{\prime}$ & 2414 & $12 / 07 / 07$ & Bottom water sampler \\
\hline ARKXXII/1c & PS70/174-1 & HGIV & $78^{\circ} 36.54^{\prime}$ & $5^{\circ} 3.82^{\prime}$ & 2354 & $13 / 07 / 07$ & Multiple corer \\
\hline ARKXXII/1c & PS70/175-1 & HGIV & $78^{\circ} 46.85^{\prime}$ & $5^{\circ} 19.98^{\prime}$ & 2477 & $14 / 07 / 07$ & Multiple corer \\
\hline ARKXXII/1C & PS70/183-1 & HGV & $79^{\circ} 3.92^{\prime}$ & $3^{\circ} 41.86^{\prime}$ & 2990 & $15 / 07 / 07$ & Multiple corer \\
\hline ARKXXII/1c & PS70/184-1 & HGVI & $79^{\circ} 3.60^{\prime}$ & $3^{\circ} 34.81^{\prime}$ & 3548 & $15 / 07 / 07$ & Multiple corer \\
\hline ARKXXII/1c & PS70/191-2 & HGI & $79^{\circ} 7.98^{\prime}$ & $66^{\circ} .73^{\prime}$ & 1292 & $18 / 07 / 07$ & Chamber lander \\
\hline ARKXXII/1C & PS70/206-1 & HGIV & $79^{\circ} 5.77^{\prime}$ & $4^{\circ} 10.45^{\prime}$ & 2400 & $20 / 07 / 07$ & Chamber lander \\
\hline ARKXXII/1c & PS70/211-1 & HGVII & $79^{\circ} 3.59^{\prime}$ & $3^{\circ} 28.50^{\prime}$ & 4065 & $19 / 07 / 07$ & Multiple corer \\
\hline ARKXXII/1c & PS70/212-1 & HGVIII & $79^{\circ} 3.79^{\prime}$ & $3^{\circ} 18.80^{\prime}$ & 5140 & $19 / 07 / 07$ & Multiple corer \\
\hline ARKXXII/1c & PS70/219-1 & HGIX & $79^{\circ} 7.89^{\prime}$ & $2{ }^{\circ} 50.23^{\prime}$ & 5586 & $22 / 07 / 07$ & Chamber lander \\
\hline
\end{tabular}

onto pre-combusted Whatman GF/F filters and treated in the same way as above.

To obtain the $\delta^{15} \mathrm{~N}$ of deposit feeders' food source (sediments, organic matter and associated organisms) bulk sediment samples from the uppermost $5 \mathrm{~cm}$ were collected with a multiple corer, the benthic chambers of a lander and a bottom water sampler at different depths in summer 2007 (Table 1). Sediment samples and biota were stored at $-80{ }^{\circ} \mathrm{C}$ until they were freeze-dried $(\geqslant 4 \mathrm{~h})$ and thereafter stored in an exsiccator. The ground samples were treated with $1 \mathrm{moll}^{-1}$ hydrochloric acid to remove carbonates (Jacob et al., 2005), re-dried at $60^{\circ} \mathrm{C}$ and ground to a fine powder by mortar and pestle.

To obtain megafaunal samples a 30 -min tow was made at a speed of 1-1.5 knots with an Agassiz trawl $(315 \mathrm{~cm}$ beam width, $110 \mathrm{~cm}$ beam height, $10 \mathrm{~kg}$ weight attached to cod-end of $20 \mathrm{~mm}$ mesh size) at HAUSGARTEN I-VII in 2004 and 2005 (Table 1). During sorting of the catch, all fish and megafauna were stored on ice. Where possible, muscle tissue of $\geqslant 5$ megafaunal individuals per taxon caught were sampled with care taken to avoid bones, intestines and gonads. However, small macrofaunal organisms had to be sampled whole and sometimes pooled to obtain sufficient biomass for isotopic analysis (Table 2). A reference sample was preserved in a $4 \%$ formaldehyde solution for identification by different taxonomic experts (see acknowledgements).

Furthermore, two fish traps fitted to the tripod of an autonomous benthic lander were deployed for $\sim 48 \mathrm{~h}$. A baited funnel trap was deployed within each fish trap to prevent the fish from feeding on fish bait and to separate them from trapped amphipods. All fish and amphipods were sampled as described above. More samples were collected from $R V$ L'Atalante in September 2005 with the hydraulic slurp gun of the remotely operated vehicle 'Victor 6000' at HAUSGARTEN IV and IX (Table 1).

Stable isotope analysis was done by isotope-ratio mass spectrometry (Thermo/Finnigan Delta Plus at GeoBioCenterLMU, University of Munich). Gaseous standards ( $\mathrm{N}_{2}$ and $\mathrm{CO}_{2}$, respectively) were calibrated against atmospheric nitrogen (AIR) and a PeeDee Belemite standard (PDB) for carbon. Analytical error was $\pm 0.15 \%$ based on the standard deviation of replicates of a peptone standard.

\subsection{Data analysis}

Stable isotope ratios are expressed in the conventional $\delta$ notation as parts per thousand (\%) according to the following equation:

$\delta X=\left[\left(R_{\text {sample }} / R_{\text {standard }}\right)-1\right] \times 1000$

where $X$ is ${ }^{13} \mathrm{C}$ or ${ }^{15} \mathrm{~N}$ and $R$ is the corresponding ratio ${ }^{13} \mathrm{C} /{ }^{12} \mathrm{C}$ or ${ }^{15} \mathrm{~N} /{ }^{14} \mathrm{~N}$. We used a $\delta^{15} \mathrm{~N}$ enrichment factor of $3.8 \%$ per trophic level to keep our results comparable with those from previous Arctic food web studies (Hobson and Welch, 1992; Adlandsvik et al., 2004; Iken et al., 2005):

$\mathrm{TL}_{i}=\left(\delta^{15} \mathrm{~N}_{i}-\delta^{15} \mathrm{~N}_{\text {ref }}\right) / 3.8$

where $\mathrm{TL}_{i}$ is the trophic level of species $i, \delta^{15} \mathrm{~N}_{i}$ is the mean species $\delta^{15} \mathrm{~N}$ and $\delta^{15} \mathrm{~N}_{\text {ref }}$ is the mean $\delta^{15} \mathrm{~N}$ of the food web baseline (POM). 
Table 2

Mean stable isotope signatures of taxa from different depths..

\begin{tabular}{|c|c|c|c|c|c|c|c|c|c|c|}
\hline Taxonomic group & Taxon & Station No. & Depth (m) & Sampling & Feeding type & $n$ & $\delta^{13} \mathrm{C}(\%)$ & SEM & $\delta^{15} \mathrm{~N}(\%)$ & SEM \\
\hline Foraminifera & Foraminifera & PS68/276-1 & 1500 & 7 whole ind. & n.d. & 2 & -26.66 & 1.93 & 4.1 & 1.1 \\
\hline Hexactinellida & Caulophacus arcticus & PS66/118-1 & 2400 & Section & SF & 5 & -0.25 & 0.43 & 10.6 & 1.7 \\
\hline Hexactinellida & Caulophacus arcticus & PS68/260-1 & 3600 & Section & $\mathrm{SF}$ & 3 & -21.22 & 0.88 & 12.2 & 1.8 \\
\hline Demospongiae & Axinellidae & PS68/240-1 & 2400 & Section & $\mathrm{SF}$ & 2 & -23.02 & 0.12 & 7.84 & 0 \\
\hline Demospongiae & Cladorhiza gelida & PS68/272-2 & 1900 & Section & $\mathrm{SF}$ & 3 & -19.5 & 0.37 & 9.12 & 0.6 \\
\hline Demospongiae & Cladorhiza gelida & PS66/118-1 & 2400 & Section & SF & 5 & -18.16 & 0.17 & 9.55 & 0.1 \\
\hline Demospongiae & Cladorhiza gelida & Victor-255 & 5400 & Section & SF & 2 & -0.33 & 0.67 & 8.57 & 0.5 \\
\hline Demospongiae & Esperiopsis sp. & PS68/276-1 & 1500 & Section & SF & 1 & -21.32 & & 11.3 & \\
\hline Demospongiae & Esperiopsis sp. & PS68/272-2 & 1900 & Section & SF & 2 & -19.46 & 1.96 & 16.6 & 0.4 \\
\hline Demospongiae & Lissodendoryx complicata & PS68/247-1 & 3100 & Section & SF & 3 & -0.48 & 0.23 & 13.7 & 0.4 \\
\hline Demospongiae & Radiella sol & PS68/247-1 & 3100 & Section & SF & 3 & -0.51 & 0.58 & 12.4 & 1.2 \\
\hline Demospongiae & Tentorium semisuberites & PS68/272-2 & 1900 & 1 whole ind. & $\mathrm{SF}$ & 4 & -21.8 & 0.52 & 13 & 0.9 \\
\hline Demospongiae & Thenea abyssorum & PS68/247-1 & 3100 & 1 whole ind. & $\mathrm{SF}$ & 5 & -0.32 & 0.15 & 12.1 & 0.8 \\
\hline \multicolumn{11}{|l|}{ Cnidaria } \\
\hline Hydrozoa & Thecate hydroid & PS68/240-1 & 2400 & Section & $\mathrm{SF}$ & 1 & -23.1 & & 7.62 & \\
\hline Alcyonacea & Gersemia rubiformis & PS68/272-2 & 1900 & Section & $\mathrm{SF}$ & 5 & -21.88 & 0.16 & 11.2 & 0.3 \\
\hline Actiniaria & Acontiaria gen. et sp. nov. & PS66/118-1 & 2400 & Septum & $\mathrm{SF}$ & 5 & -21.55 & 0.85 & 10.8 & 0.6 \\
\hline Actiniaria & Hormathiidae cf. Amphianthus sp. & PS68/256-1 & 3900 & 1 whole ind. & $\mathrm{SF}$ & 3 & -21.97 & 0.29 & 12.2 & 0.3 \\
\hline Actiniaria & Kadosactis rosea & PS68/260-1 & 3600 & Septum & $\mathrm{SF}$ & 3 & -19.1 & 0.23 & 15.3 & 0.4 \\
\hline Actiniaria & Amphianthus sp. 1 & PS68/260- 1 & 3600 & Septum & $\mathrm{SF}$ & 1 & -21.82 & & 11.4 & \\
\hline Actiniaria & Amphianthus sp. 2 & PS68/260-1 & 3600 & Septum & $\mathrm{SF}$ & 5 & -22.09 & 0.35 & 11.3 & 0.3 \\
\hline Actiniaria & Amphianthus sp. 4, Isophelliidae gen.1 & PS68/272-2 & 1900 & 4 pooled & $\mathrm{SF}$ & 2 & -22.61 & 0.29 & 9.77 & 1.8 \\
\hline Actiniaria & Amphianthus spp., Bathyphellia margaritacea & PS68/247-1 & 3100 & Septum & $\mathrm{SF}$ & 4 & -0.69 & 0.26 & 12.7 & 0.5 \\
\hline Actiniaria & Bathyphellia margaritacea & PS68/260-1 & 3600 & Septum & $\mathrm{SF}$ & 9 & -21.44 & 0.16 & 11.2 & 0.2 \\
\hline Actiniaria & Bathyphellia margaritacea & PS68/256-1 & 3900 & 2 whole ind. & $\mathrm{SF}$ & 7 & -21.92 & 0.25 & 11.9 & 0.2 \\
\hline Actiniaria & Bathyphellia margaritacea & Victor-255 & 5400 & Septum & $\mathrm{SF}$ & 5 & -19.61 & 0.26 & 16.6 & 0.2 \\
\hline Actiniaria & Isophelliidae gen. 1 & PS68/272-2 & 1900 & Septum & $\mathrm{SF}$ & 2 & -0.83 & 0.03 & 12.8 & 0.1 \\
\hline Errect biota & Hydroid or sponge & PS68/260-1 & 3600 & Section & SF & 1 & -21.61 & & 8.5 & \\
\hline Nemertea & Nemertea & PS68/276-1 & 1500 & Posterior part & $\mathrm{P} / \mathrm{S}$ & 4 & -19.01 & 0.67 & 12.4 & 2 \\
\hline Priapulidae & Priapulus caudatus & PS68/276-1 & 1500 & Section & DF & 1 & -17.17 & & 13.8 & \\
\hline
\end{tabular}




\begin{tabular}{|c|c|c|c|c|c|c|c|c|c|c|}
\hline \multicolumn{11}{|l|}{ Mollusca } \\
\hline Gastropoda & Cryptonatica affinis & PS68/276-1 & 1500 & 1 whole ind. & DF & 2 & -19.06 & 0.65 & 9.7 & 0.3 \\
\hline Gastropoda & Mohnia mohni, Tacita danielsseni & PS66/103-1 & 1300 & Section of foot & $\mathrm{P} / \mathrm{S}$ & 2 & -18.22 & 0.02 & 13.4 & 0.5 \\
\hline Gastropoda & Mohnia mohni, Tacita danielsseni & PS68/276-1 & 1500 & Section of foot & $\mathrm{P} / \mathrm{S}$ & 2 & -18.95 & 0.12 & 10.9 & 0.2 \\
\hline Gastropoda & Mohnia mohni, Tacita danielsseni & PS68/272-2 & 1900 & Section of foot & $\mathrm{P} / \mathrm{S}$ & 6 & -0.02 & 0.11 & 10.7 & 0.2 \\
\hline Gastropoda & Mohnia mohni, Tacita danielsseni & PS66/118-1 & 2400 & Section of foot & $\mathrm{P} / \mathrm{S}$ & 5 & -17.99 & 0.33 & 13.2 & 0.1 \\
\hline Gastropoda & Mohnia mohni, Tacita danielsseni & PS68/247-1 & 3100 & Section of foot & $\mathrm{P} / \mathrm{S}$ & 5 & -19.69 & 0.33 & 10.6 & 0.7 \\
\hline Bivalvia & Bathyarca frielei & PS68/276-1 & 1500 & 2 whole ind. & $\mathrm{SF}$ & 3 & -0.08 & 0.7 & 10.9 & 1.3 \\
\hline Bivalvia & Katadesmia kolthoffi & PS68/260-1 & 3600 & 1 whole ind. & $\mathrm{DF}$ & 8 & -0.4 & 0.16 & 8.29 & 0.5 \\
\hline Bivalvia & Katadesmia kolthoffi & PS68/256-1 & 3900 & 1 whole ind. & $\mathrm{DF}$ & 2 & -19.7 & 0.03 & 10.5 & 0.1 \\
\hline Scaphopoda & Siphonodentalium laubieri & PS68/276-1 & 1500 & $3-4$ whole ind. & $\mathrm{DF}$ & 5 & -0.64 & 0.08 & 10.8 & 0.1 \\
\hline \multicolumn{11}{|l|}{ Annelida } \\
\hline Polychaeta & Bylgides cf. groenlandica & PS68/272-2 & 1900 & Posterior part & $\mathrm{P} / \mathrm{S}$ & 2 & -19.76 & 0.21 & 11.8 & 0.5 \\
\hline Polychaeta & Lumbrineris sp. & PS68/276-1 & 1500 & Posterior part & n.d. & 1 & -19.81 & & 12.5 & \\
\hline Polychaeta & Nereis cf. gracilis & PS68/272-2 & 1900 & Posterior part & $\mathrm{P} / \mathrm{S}$ & 1 & -17.97 & & 15.8 & \\
\hline Polychaeta & Praxillura longissima & PS68/276-1 & 1500 & 1 whole ind. & $\mathrm{DF}$ & 1 & -21.3 & & 15.1 & \\
\hline Polychaeta & Polychaeta sp. 1 & PS68/247-1 & 3100 & 1 whole ind. & n.d. & 1 & -16.98 & & 14.4 & \\
\hline Polychaeta & Polychaeta sp. 2 & PS68/260-1 & 3600 & 1 whole ind. & n.d. & 1 & -18.57 & & 15.2 & \\
\hline Polychaeta & Polychaeta sp. 3 & PS68/272-2 & 1900 & 1 whole ind. & n.d. & 1 & -22.69 & & 7.74 & \\
\hline Polychaeta & Polychaeta sp. 4 & PS68/276-1 & 1500 & Posterior part & n.d. & 1 & -20.54 & & 9.74 & \\
\hline Polychaeta & Polychaeta sp. 5 & PS68/272-2 & 1900 & 3 whole ind. & n.d. & 8 & -19.53 & 0.18 & 10.8 & 0.1 \\
\hline Polychaeta & Polychaeta sp. 6 & PS68/276-1 & 1500 & 1 whole ind. & n.d. & 2 & -20.78 & 0.04 & 11.1 & 0 \\
\hline Echiurida & Hamingia arctica & PS66/103-1 & 1300 & Body wall of 1 & $\mathrm{DF}$ & 5 & -18.01 & 1.07 & 12.9 & 0.8 \\
\hline Echiurida & Hamingia arctica & PS68/276-1 & 1500 & Body wall of 1 & $\mathrm{DF}$ & 2 & -16.04 & 0.58 & 14.7 & 0.1 \\
\hline \multicolumn{11}{|l|}{ Arthropoda } \\
\hline Pantopoda & Ascorhynchus abyssi & PS68/260-1 & 3600 & 1 whole ind. & DF & 1 & -21.55 & & 9.65 & \\
\hline Pantopoda & Colossendeis proboscidea & PS66/103-1 & 1300 & 4 legs of various ind. & $\mathrm{DF}$ & 3 & -18.04 & 0.03 & 12.2 & 0.1 \\
\hline Pantopoda & Colossendeis proboscidea & PS68/276-1 & 1500 & 4 legs of various ind. & $\mathrm{DF}$ & 2 & $\begin{array}{l}-19.71 \\
-\end{array}$ & 1.83 & 9.52 & 2.7 \\
\hline Copepoda & Calanoida & Victor-256 & 2400 & 1 whole ind. & HBPF & 2 & -0.09 & 0.74 & 10.7 & 1.6 \\
\hline Cirripedia & Verum striolatum & PS68/276-1 & 1500 & M & SF & 2 & -21.45 & 0.13 & 11.3 & 0.1 \\
\hline Cirripedia & Verum striolatum & PS68/272-2 & 1900 & $\mathrm{M}$ & $\mathrm{SF}$ & 1 & -22.18 & & 10 & \\
\hline Mysida & Boreomysis sp. & PS68/247-1 & 3100 & Abdomen of 1 & HBPF & 1 & -0.58 & & 14.2 & \\
\hline Amphipoda & Corophiidae & PS68/276-1 & 1500 & 1 whole ind. & n.d. & 1 & -21.18 & & 9.15 & \\
\hline Amphipoda & Eurythenes gryllus & PS68/278-1 & 2400 & Gutted telson of 1 & $\mathrm{P} / \mathrm{S}$ & 8 & -22.08 & 0.12 & 14.8 & 0.6 \\
\hline Amphipoda & Eurythenes gryllus & PS68/247-1 & 3100 & Gutted telson of 1 & $\mathrm{P} / \mathrm{S}$ & 3 & -22.76 & 0.37 & 12.8 & 0.4 \\
\hline Amphipoda & Eurythenes gryllus & PS68/270-1 & 3600 & Gutted telson of 1 & $\mathrm{P} / \mathrm{S}$ & 4 & -21.65 & 0.33 & 12.7 & 0.7 \\
\hline Amphipoda & Halirages quadridentatus & Victor-255 & 5400 & Gutted telson of 1 & $\mathrm{P} / \mathrm{S}$ & 6 & -21.31 & 0.15 & 10 & 0.2 \\
\hline Amphipoda & Haploops sp. & PS68/272-2 & 1900 & 1 whole ind. & $\mathrm{SF}$ & 6 & -22.14 & 0.14 & 8.7 & 1 \\
\hline Amphipoda & Leptamphopus sp. & Victor-256 & 2400 & 3 whole ind. & $\mathrm{DF}$ & 2 & -0.99 & 0.15 & 7.63 & 0.5 \\
\hline Amphipoda & Liljeborgia fissicornis & PS68/276-1 & 1500 & 1 whole ind. & $\mathrm{P} / \mathrm{S}$ & 1 & -19.75 & & 10.9 & \\
\hline Amphipoda & Lysianassidae cf. tryphosa & Victor-255 & 5400 & 3 whole ind. & $\mathrm{P} / \mathrm{S}$ & 3 & -23.36 & 0.18 & 14.2 & 0.2 \\
\hline Amphipoda & Amphipoda sp. 1 & PS68/272-2 & 1900 & 1 whole ind. & n.d. & 2 & -21.89 & 0.95 & 8.41 & 0.9 \\
\hline Amphipoda & Amphipoda sp. 2 & PS68/276-1 & 1500 & 4 whole ind. & n.d. & 1 & -21.54 & & 6.08 & \\
\hline Amphipoda & Amphipoda sp. 3 & PS68/272-2 & 1900 & 1 whole ind. & n.d. & 1 & -21.11 & & 7.64 & \\
\hline Amphipoda & Unciola sp. & PS68/276-1 & 1500 & 2 whole ind. & $\mathrm{DF}$ & 2 & -21.14 & 0.06 & 5.63 & 0.7 \\
\hline Cumacea & Diastylis spp. & PS68/276-1 & 1500 & 1 whole ind. & $\mathrm{DF}$ & 3 & -21.59 & 0.16 & 5.4 & 0.3 \\
\hline Isopoda & Caecognathia stygia & PS68/276-1 & 1500 & 3 whole ind. & $\mathrm{DF}$ & 1 & -21.94 & & 9.72 & \\
\hline
\end{tabular}




\begin{tabular}{|c|c|c|c|c|c|c|c|c|c|c|}
\hline Taxonomic group & Taxon & Station No. & Depth $(\mathrm{m})$ & Sampling & Feeding type & $n$ & $\delta^{13} \mathrm{C}(\%)$ & SEM & $\delta^{15} \mathrm{~N}(\%)$ & SEM \\
\hline Isopoda & Caecognathia stygia & PS68/272-2 & 1900 & 1 whole ind. & DF & 2 & -22.08 & 0.04 & 10.4 & 0.5 \\
\hline Isopoda & Mesidothea megalura & PS68/276-1 & 1500 & Abdomen of 1 & $\mathrm{P} / \mathrm{S}$ & 3 & -0.19 & 0.03 & 10.7 & 0.2 \\
\hline Isopoda & Mesidothea megalura & PS68/272-2 & 1900 & Abdomen of 1 & $\mathrm{P} / \mathrm{S}$ & 8 & -0.07 & 0.28 & 10.1 & 0.4 \\
\hline Isopoda & Mesidothea megalura & PS66/118-1 & 2400 & Abdomen & $\mathrm{P} / \mathrm{S}$ & 8 & -19.59 & 0.35 & 11.3 & 0.5 \\
\hline Isopoda & Mesidothea megalura & PS68/247-1 & 3100 & Abdomen of 1 & $\mathrm{P} / \mathrm{S}$ & 1 & -19.58 & & 10.5 & \\
\hline Decapoda & Bythocaris spp. & PS66/103-1 & 1300 & Gutted abdomen of 1 & $\mathrm{P} / \mathrm{S}$ & 5 & -18.26 & 0.08 & 15.2 & 0.2 \\
\hline Decapoda & Bythocaris spp. & PS68/272-2 & 1900 & Gutted abdomen of 1 & $\mathrm{P} / \mathrm{S}$ & 6 & -19.62 & 0.17 & 13 & 0.2 \\
\hline Decapoda & Bythocaris spp. & PS66/118-1 & 2400 & Gutted abdomen of 1 & $\mathrm{P} / \mathrm{S}$ & 5 & -19.41 & 0.24 & 13.2 & 0.2 \\
\hline Decapoda & Bythocaris spp. & PS68/260-1 & 3600 & Gutted abdomen of 1 & $\mathrm{P} / \mathrm{S}$ & 6 & -19.7 & 0.19 & 13.8 & 0.1 \\
\hline Decapoda & Bythocaris spp. & PS68/256-1 & 3900 & Gutted abdomen of 1 & $\mathrm{P} / \mathrm{S}$ & 3 & -0.49 & 0.07 & 13.8 & 0.1 \\
\hline \multicolumn{11}{|l|}{ Echinodermata } \\
\hline Crinoidea & Bathycrinus cf. carpenteri & PS68/272-2 & 1900 & 3 pooled & $\mathrm{SF}$ & 3 & -23.12 & 0.22 & 8.28 & 0.9 \\
\hline Crinoidea & Bathycrinus cf. carpenteri & PS66/118-1 & 2400 & 5 pooled & $\mathrm{SF}$ & 5 & -22.17 & 0.25 & 10.4 & 0.7 \\
\hline Crinoidea & Bathycrinus cf. carpenteri & PS68/247-1 & 3100 & 5 pooled & $\mathrm{SF}$ & 1 & -22.84 & & 11 & \\
\hline Asteroidea & Bathybiaster vexillifer & PS66/103-1 & 1300 & Arm of 1 & $\mathrm{P} / \mathrm{S}$ & 5 & -17.1 & 0.2 & 15.8 & 0.1 \\
\hline Asteroidea & Bathybiaster vexillifer & PS68/276-1 & 1500 & 2 arms of 1 & $\mathrm{P} / \mathrm{S}$ & 8 & -17.08 & 0.18 & 16.8 & 0.3 \\
\hline Asteroidea & Bathybiaster vexillifer & PS68/272-2 & 1900 & Arm of 1 & $\mathrm{P} / \mathrm{S}$ & 2 & -17.05 & 0.62 & 17 & 0.4 \\
\hline Asteroidea & Hymenaster pellucidus & PS68/276-1 & 1500 & 3 arms of 1 & $\mathrm{P} / \mathrm{S}$ & 3 & -19.49 & 0.24 & 13.1 & 0.7 \\
\hline Asteroidea & Hymenaster pellucidus & PS68/272-2 & 1900 & Arm of 1 & $\mathrm{P} / \mathrm{S}$ & 3 & -19.89 & 0.11 & 11.9 & 0.7 \\
\hline Asteroidea & Hymenaster pellucidus & PS66/118-1 & 2400 & $1 \mathrm{arm}$ & $\mathrm{P} / \mathrm{S}$ & 1 & -19.54 & & 24.6 & \\
\hline Asteroidea & Poraniomorpha tumida & PS68/247-1 & 3100 & Arm of 1 & $\mathrm{P} / \mathrm{S}$ & 4 & -17.27 & 0.7 & 21.3 & 0.4 \\
\hline Ophiuroidea & Ophiocten cf. gracilis & PS66/103-1 & 1300 & $15 \mathrm{arms}$ of various ind. & $\mathrm{DF}$ & 5 & -0.14 & 0.35 & 12.6 & 0.3 \\
\hline Ophiuroidea & Ophiocten cf. gracilis & PS68/276-1 & 1500 & $5 \mathrm{arms}$ & DF & 6 & -0.49 & 0.12 & 11 & 0.6 \\
\hline Holothuroidea & Kolga hyalina & PS68/247-1 & 3100 & Body wall of 1 & DF & 3 & -0.26 & 0.24 & 8.87 & 0.3 \\
\hline Holothuroidea & Elpidia heckeri & Victor-255 & 5400 & Body wall of 1 & DF & 2 & -22.24 & 0.91 & 5.98 & 0.9 \\
\hline \multicolumn{11}{|l|}{ Chordata } \\
\hline Ascidiacea & Ascidiacea & PS68/276-1 & 1500 & 1 whole ind. & SF & 1 & -18.93 & & 13.1 & \\
\hline Rajiformes & Amblyraja hyperborea & PS66/103-1 & 1300 & $\mathrm{~m}$ & $\mathrm{P} / \mathrm{S}$ & 1 & -0.36 & & 12.4 & \\
\hline Teleostei & Gaidropsarus argentatus & PS66/103-1 & 1300 & $\mathrm{~m}$ & $\mathrm{P} / \mathrm{S}$ & 1 & -19.97 & & 13 & \\
\hline Teleostei & Lycodes frigidus & PS68/272-2 & 1900 & $\mathrm{~m}$ & $\mathrm{P} / \mathrm{S}$ & 6 & -19.3 & 0.12 & 13.6 & 0.1 \\
\hline Teleostei & Lycodes frigidus & Victor-256 & 2400 & $\mathrm{~m}$ & $\mathrm{P} / \mathrm{S}$ & 23 & -0.66 & 0.2 & 12.4 & 0.2 \\
\hline Teleostei & Lycodes frigidus & PS68/247-2 & 3100 & $\mathrm{~m}$ & $\mathrm{P} / \mathrm{S}$ & 3 & -19.34 & 0.29 & 14.1 & 0.2 \\
\hline Teleostei & Lycodes frigidus & PS68/260-1 & 3600 & $\mathrm{~m}$ & $\mathrm{P} / \mathrm{S}$ & 1 & -19.83 & & 14.6 & \\
\hline Teleostei & Lycodes squamiventer & PS66/103-1 & 1300 & $\mathrm{~m}$ & $\mathrm{P} / \mathrm{S}$ & 12 & -18.59 & 0.06 & 13.9 & 0.1 \\
\hline Teleostei & Lycodes squamiventer & PS68/276-1 & 1500 & $\mathrm{~m}$ & $\mathrm{P} / \mathrm{S}$ & 5 & -18.74 & 0.23 & 13.3 & 0.3 \\
\hline Teleostei & Lycodonus flagellicauda & PS68/276-1 & 1500 & $\mathrm{~m}$ & $\mathrm{P} / \mathrm{S}$ & 2 & -19.92 & 0.14 & 13 & 0.6 \\
\hline Teleostei & Paralepididae & PS68/272-2 & 1900 & $\mathrm{~m}$ & Decomposing & 1 & -22.39 & & 10.8 & \\
\hline Cetacea & Whale bone & Victor-255 & 5400 & Section & Decomposed & 6 & -16.18 & 0.17 & 10.2 & 0.1 \\
\hline
\end{tabular}




\begin{tabular}{|c|c|c|c|c|c|c|c|c|}
\hline \multirow[t]{18}{*}{ Food sources } & POM (surface) & PS68/245-1 & 10 & Filtrate & 1 & n.a. & 6.03 & \\
\hline & POM (surface) & PS68/245-1 & 20 & Filtrate & 1 & n.a. & 4.66 & \\
\hline & POM (surface) & PS68/245-1 & 30 & Filtrate & 1 & n.a. & 5.1 & \\
\hline & POM (surface) & PS68/245-1 & 50 & Filtrate & 1 & n.a. & 4.55 & \\
\hline & POM (bottom) & PS66/134-1 & 2400 & Filtrate & 13 & n.a. & 4.43 & 0.2 \\
\hline & POM (bottom) & PS64/399-1 & 2200 & Filtrate & 19 & n.a. & 4.43 & 0.3 \\
\hline & Sediment & PS70/191-2 & 1292 & Chamber & 3 & n.a. & 5.28 & \\
\hline & Sediment & PS70/163-1 & 1304 & Multiple corer & 3 & n.a. & 5.69 & 0.28 \\
\hline & Sediment & PS70/159-1 & 1565 & Multiple corer & 3 & n.a. & 5.52 & 0.04 \\
\hline & Sediment & PS70/174-1 & 2354 & Multiple corer & 1 & n.a. & 5.86 & \\
\hline & Sediment & PS70/206-1 & 2400 & Chamber & 3 & n.a. & 5.26 & 0.13 \\
\hline & Sediment & PS70/169-1 & 2414 & BWS & 3 & n.a. & 5.43 & 0.02 \\
\hline & Sediment & PS70/200-1 & 2644 & Multiple corer & 3 & n.a. & 5.58 & 0.04 \\
\hline & Sediment & PS70/183-1 & 2990 & Multiple corer & 3 & n.a. & 5.21 & 0.05 \\
\hline & Sediment & PS70/184-1 & 3593 & Multiple corer & 3 & n.a. & 4.85 & 0.03 \\
\hline & Sediment & PS70/211-1 & 4065 & Multiple corer & 3 & n.a. & 4.77 & 0.07 \\
\hline & Sediment & PS70/212-1 & 5140 & Multiple corer & 3 & n.a. & 4.22 & 0.07 \\
\hline & Sediment & PS70/219-1 & 5586 & Chamber & 3 & n.a. & 5.41 & 0.04 \\
\hline
\end{tabular}

Abbreviations: (SEM) standard error of the mean, (P/S) Predator/scavenger, (SF) suspension feeder, (DF) deposit feeder, (HBPF) hyperbenthic particle feeder, (n.d.) not defined, (n.a.) not available, (M) muscle tissue, (POM) particulate organic matter, (ind.) individual, (BWS) bottom water sampler. 
Overall, the stable nitrogen and carbon content of 73 taxa, was analysed (430 samples in total, including POM and sediment samples). Based on information from the literature and advice from specialists, organisms were assigned to one of the following functional groups: predator/scavenger, suspension feeder, deposit feeder. If the taxonomic resolution was too low, or if no trophic information was available, the organisms were excluded from this analysis.

We tested for significant differences in the $\delta^{15} \mathrm{~N}$ signatures of different functional groups, phyla and organisms collected from different depths using analysis of variance (ANOVA) and Tukey's pairwise comparisons. We applied linear regression analysis to test if the $\delta^{15} \mathrm{~N}$ signatures of organisms varied as a function of depth. For all statistical analyses we used the MINITAB $14^{\circ}$ package.

\section{Results}

\subsection{General food web structure}

The $\delta^{15} \mathrm{~N}$ values of POM from the euphotic zone were higher (mean $\delta^{15} \mathrm{~N}=5.4 \pm 0.5 \%$ sEM) than those recorded at $800 \mathrm{~m}$ depth $(4.6 \pm 0.3 \%)$ and at the sea floor $\left(4.4 \pm 0.2 \%\right.$ ). The $\delta^{15} \mathrm{~N}$ ratios of POM collected by sediment traps deployed at $800 \mathrm{~m}$ and above the sea bed were similar (ANOVA: $F_{(2,49)}=0.16, p=0.85$ ). The $\delta^{15} \mathrm{~N}$ values of sediments were slightly higher than most POM samples (Table 2). We used the mean $\delta^{15} \mathrm{~N}$ values of POM collected by bottom sediment traps as a baseline as we consider this a key source of food for benthic organisms.

There was a considerable range of $\delta^{15} \mathrm{~N}$ values in the food web sampled (Table 2): it ranged from $3.0 \%$ or Foraminifera to $21.4 \%$ for starfish (Poraniomorpha tumida).

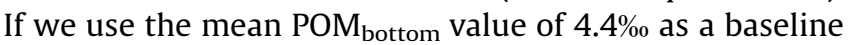
and assume a progressive $\delta^{15} \mathrm{~N}$ enrichment of $3.8 \%$ per trophic step, the HAUSGARTEN food web encompassed five trophic levels (Fig. 2).

While the majority of deposit feeders (15 taxa) occupied the first and second trophic level (10.2 $\pm 0.3 \%$ ), most suspension feeders (25 taxa) fed at the second and third $(11.2 \pm 0.2 \%$ ) and most predators/scavengers (19 taxa) occupied the third trophic level $(13.2 \pm 0.2 \%$ ) (Fig. 2). There were significant differences in the $\delta^{15} \mathrm{~N}$ signatures between each of these groups (ANOVA, $\left.F_{(2,358)}=43.74, p<0.001\right)$.

Predators/scavengers had the highest range of $\delta^{15} \mathrm{~N}$ values $(13.5 \%$ ) followed by suspension (11.4\%o) and deposit $(10.1 \%$ ) feeders.

All organisms except for Foraminifera were enriched in $\delta^{15} \mathrm{~N}$ relative to POM (Table 2). Poraniomorpha tumida was the only species to occupy the fifth trophic level with the highest mean $\delta^{15} \mathrm{~N}$ recorded $(21.4 \pm 0.4 \%$ ) followed by the anthozoan Bathyphellia margaritacea and the starfish Bathybiaster vexillifer (Table 2). The majority of organisms occupied the second and third trophic levels (Fig. 2). Cumaceans (Diastylis spp.), amphipods and hydrozoans had the lowest mean $\delta^{15} \mathrm{~N}$ (first trophic level). Molluscs occupied exclusively the second, and fish and rays occupied the third trophic level.
Table 2 shows that there was great variability in taxon/ species isotopic enrichment: the range of $\delta^{15} \mathrm{~N}$ values exceeded two trophic levels in nemerteans and the sponge Caulophacus arcticus and one level in Eurythenes gryllus, Haploops sp., Mesidothea megalura, Amphianthus spp., Colossendeis proboscidea, Gersemia rubiformis, Mohnia spp., Esperiopsis sp., Thenea abyssorum, Radiella sol, Tentorium semisuberites, Bathycrinus cf. carpenteri, Hamingia arctica, Bathyarca frielei, Katadesmia kolthoffi, Ophiocten cf. gracilis and Kolga hyalina.

\subsection{Bathymetric patterns}

Regression analysis revealed that the $\delta^{15} \mathrm{~N}$ signatures of bottom sediments decreased with increasing water depth (Fig. 3). By contrast, the $\delta^{15} \mathrm{~N}$ signatures of all organisms taken together were not affected by depth (Fig. 4a). However, analyses of different feeding types indicate that the $\delta^{15} \mathrm{~N}$ signatures of suspension feeders increased with increasing depth (Fig. 4c). Increasing numbers of carnivorous sponges have been discovered recently, some of which are closely related to those sampled here. If sponges were excluded, however, the increase of $\delta^{15} \mathrm{~N}$ of suspension feeders with depth was even more pronounced $\left(\delta^{15} \mathrm{~N}_{\mathrm{SF}}=6.95+0.00147\right.$ depth, $\left.n=81, r^{2}=0.38, p<0.0001\right)$. The $\delta^{15} \mathrm{~N}$ of sponges alone was not affected by depth $\left(\delta^{15} \mathrm{~N}_{\text {sponges }}=11.87-0.00021\right.$ depth, $\left.n=43, r^{2}=0.005, p<0.0001\right)$. By contrast, the $\delta^{15} \mathrm{~N}$ signatures of deposit feeders decreased as a function of depth (Fig. 4d). In turn, there was no significant relationship between depth and the $\delta^{15} \mathrm{~N}$ of predators/ scavengers (Fig. 4d). The low $r^{2}$-values indicate a poor regression fit.

The $\delta^{15} \mathrm{~N}$ values of most species/taxa tested were not affected by depth. However, the $\delta^{15} \mathrm{~N}$ of $B$. margaritacea and $B$. vexillifer increased significantly with increasing depth, whereas they showed a significant decrease with increasing depth in E. gryllus (Fig. 5a, c, and i).

\section{Discussion}

Naturally occurring stable nitrogen isotopes have been used widely as tracers in marine food webs as they produce estimates of trophic position that simultaneously capture trophic interactions of the reticulate pathways of communities (Hobson and Wassenaar, 1999). The popularity of the approach to assessing individual species' ecology is due to the fact that conventional tools for dietary analyses are rather limited. While it cannot provide the taxonomic detail of stomach contents analysis and direct observation, it is less time-consuming, gives information on long-term feeding habits and organisms that macerate their prey beyond recognition (Sherwood and Rose, 2005). Although recent years have seen a considerable research effort in food web ecology using this method, few studies have considered depthrelated changes in food webs. Our results indicate that most functional groups are affected by depth in a different way. 


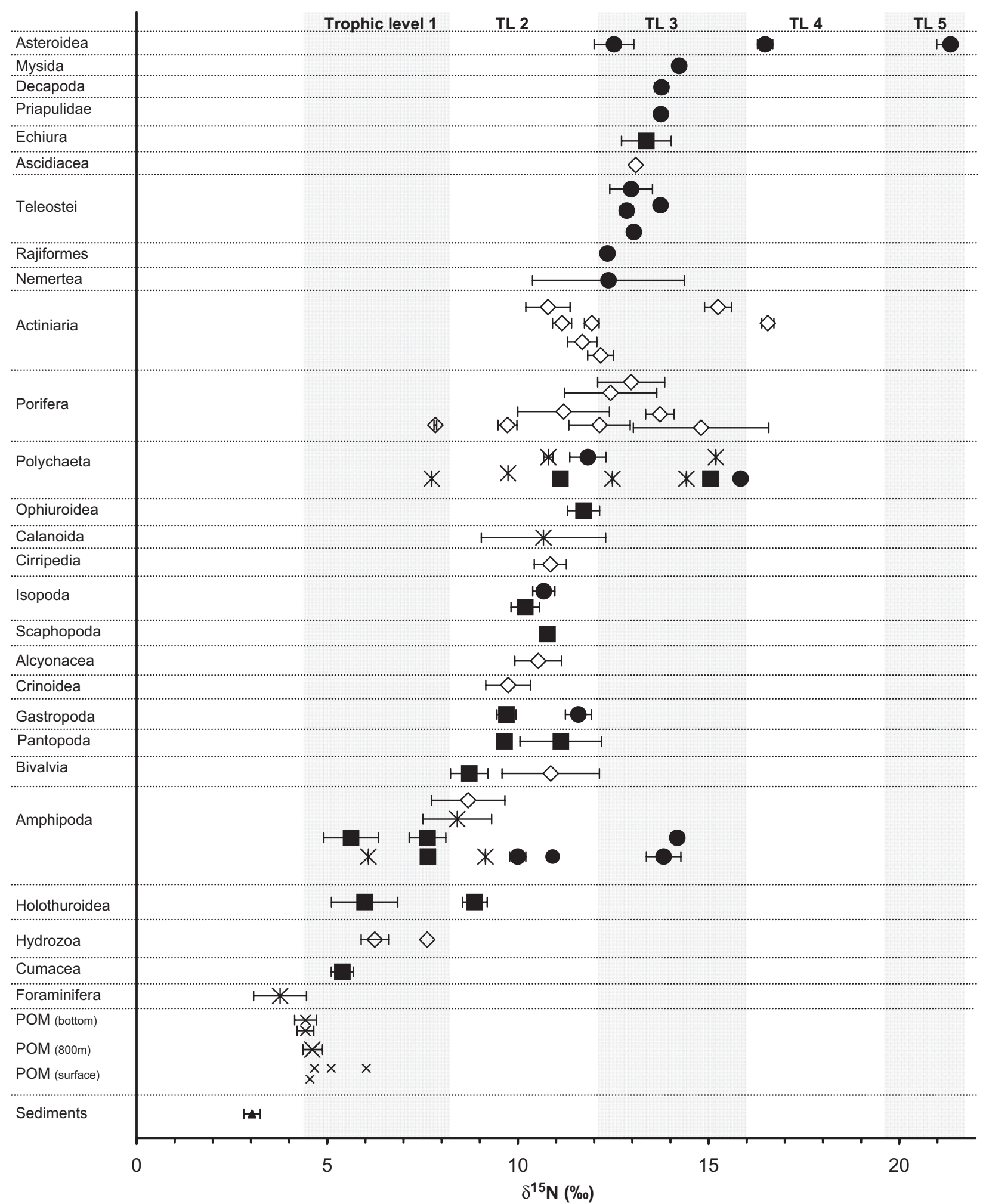

Fig. 2. Distribution of mean $\delta^{15} \mathrm{~N}$ signatures of sediments, POM and organisms from HAUSGARTEN by taxonomic groups. Trophic levels are indicated by grey shading in 3.8\% enrichment steps using bottom POM (2462 and $2393 \mathrm{~m}$ ) as a baseline. Bars indicate standard error. Symbols denote feeding types: $(\aleph i)$ not defined, $(\bullet)$ predator/scavenger, $(\diamond)$ suspension feeder and $(\boldsymbol{\square})$ deposit feeder.

\subsection{Characterisation of the food web}

\subsubsection{General food web structure}

It is likely that the benthic fauna at HAUSGARTEN utilises two to four different pathways of food: fresh surface-derived POM, (reworked) refractory bottom sediments and particles that trickle down-slope. Furthermore, large quantities of ice algae, particulate lithogenic and organic matter are trapped during freezing of the sea-ice in shallow waters, transported with the ice and released 
during melting processes (Nürnberg et al., 1994). It could therefore be argued that a multiple-source food web model (Søreide et al., 2006) should be used to distinguish between the qualitatively different inputs. However, in contrast to the input of sympagic (ice-related) systems, the $\delta^{15} \mathrm{~N}$ signatures of sediments were only slightly higher than those of POM. Therefore, it would be difficult to distinguish between different sources.

Aquatic food webs rarely encompass more than four to five trophic steps (Hall and Raffaelli, 1993). The food web

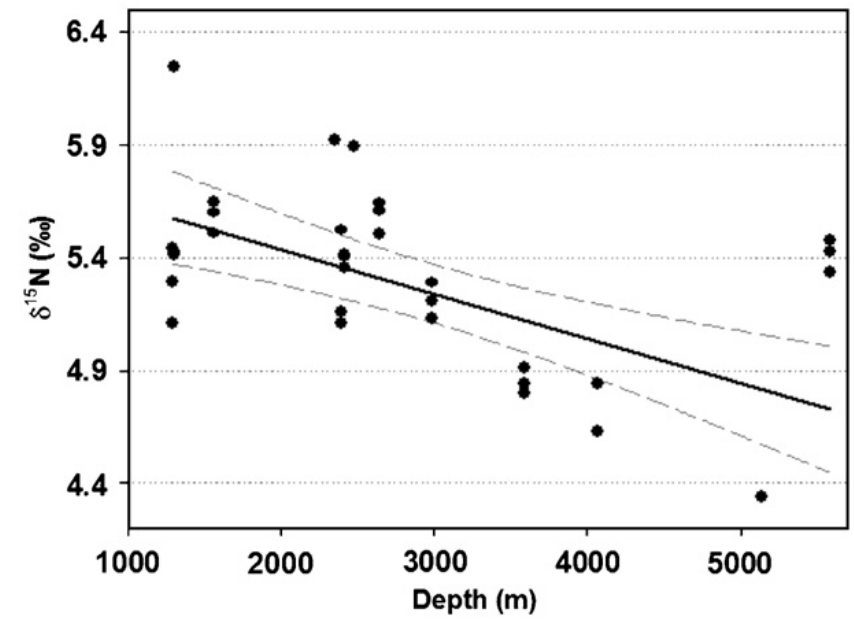

Fig. 3. Relationship between $\delta^{15} \mathrm{~N}$ signatures of sediment samples and water depth $\left(\delta^{15} \mathrm{~N}_{\mathrm{SED}}=5.833-0.000198\right.$ depth, $n=35, \quad r^{2}=0.35$, $p<0.001$ ). Dashed lines represent 95\% confidence intervals. sampled at HAUSGARTEN covered five trophic levels. While Polunin et al. (2001) reported a food chain of four trophic steps from the bathyal Mediterranean, Iken et al. (2001) found only three trophic levels at the Porcupine Abyssal Plain. Arctic food webs encompassed four trophic levels in the Canadian Arctic Basin (Iken et al., 2005), 4.7 in the Barents Sea (Tamelander et al., 2006) and five trophic levels in a Northeast Water Polynya (Hobson et al., 1995) and Lancaster Sound (Hobson and Welch, 1992). Spanning five trophic levels, the HAUSGARTEN food web clearly represents one of the longest food-chains, a situation that appears to be quite common in Arctic and deep-sea environments. Long food chain length indicates that nutrients are often recycled because of food limitation. Ecosystem size is a crucial determinant of food-chain length in aquatic systems although it is not yet clear which aspects of ecosystem size are most important (Post, 2002a). In larger ecosystems, such as those found in the deep sea (Danovaro et al., 2008), food chains might become more diverse, allowing greater dietary specialisation that could result in an increase in their lengths.

It should be noted, however, that our five trophic level estimate has to be treated with care, as it is based on the use of bottom POM as a reference. Rather than using sediments as a baseline, which would constitute chiefly a baseline of deposit feeders, we decided to use bottom POM collected by sediment traps for three reasons: (1) The sediment trap fitted to the benthic lander was suspended only 1-2 $\mathrm{m}$ above the seafloor. Therefore, it can be assumed to capture particles of various pathways (sedimenting labile POM, re-suspended refractory bottom

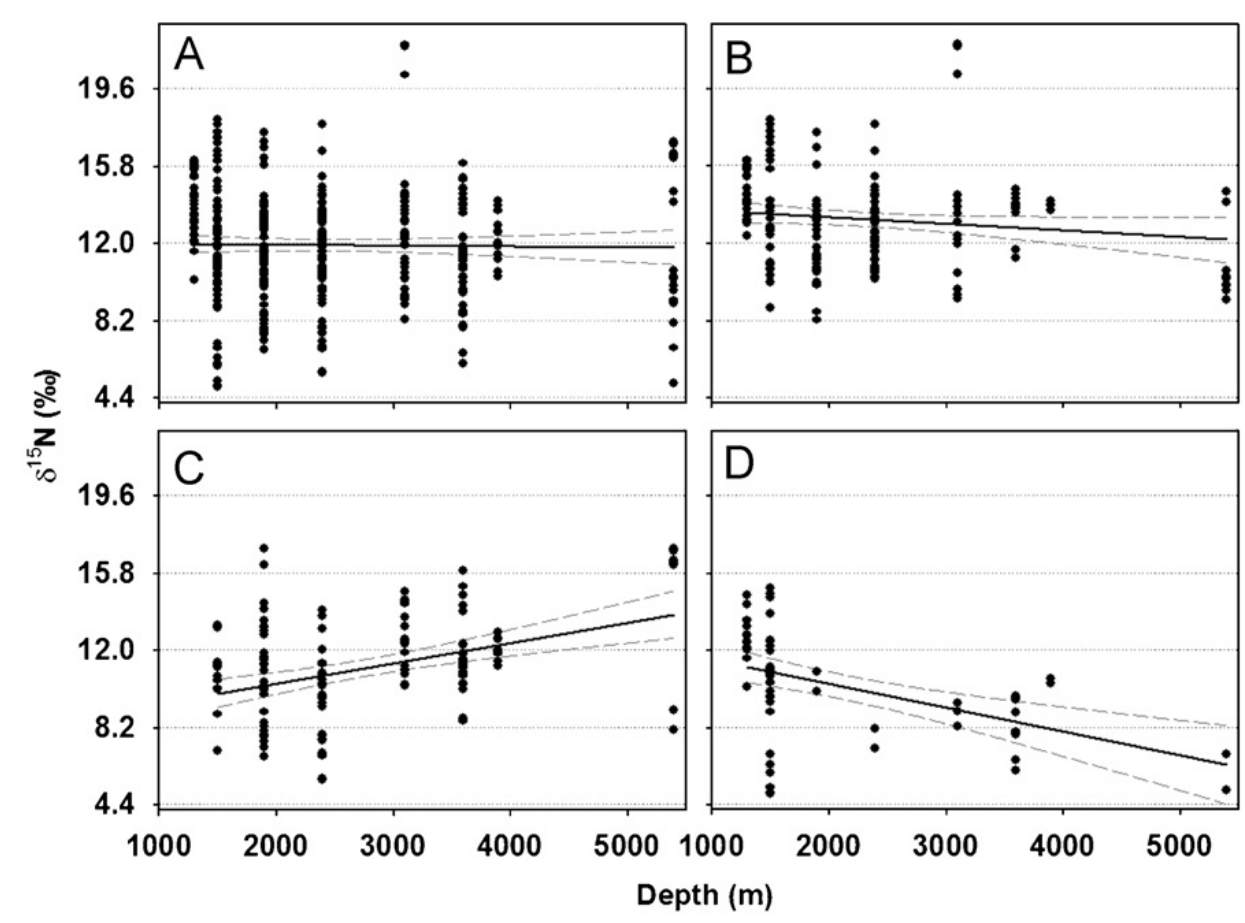

Fig. 4. Relationship between $\delta^{15} \mathrm{~N}$ signatures and water depth for (A) all organisms sampled $\left(\delta^{15} \mathrm{~N}_{\text {all }}=12-0.000039\right.$ depth, $\left.n=380, r^{2}>0.001, p=0.77\right)$, (B) predators/scavengers $\left(\delta^{15} \mathrm{~N}_{\mathrm{P} / \mathrm{S}}=13.89-0.00032\right.$ depth, $\left.n=176, r^{2}=0.019, p=0.071\right)$, (C) suspension feeders $\left(\delta^{15} \mathrm{~N}_{\mathrm{SF}}=8.386+0.00099\right.$ depth, $n=123$, $\left.r^{2}=0.15, p<0.001\right)$ and (D) deposit feeders ( $\delta^{15} \mathrm{~N}_{\mathrm{DF}}=12.64-0.00117$ depth, $\left.n=59, r^{2}=0.229, p<0.001\right)$. Dashed lines represent 95\% confidence intervals. 


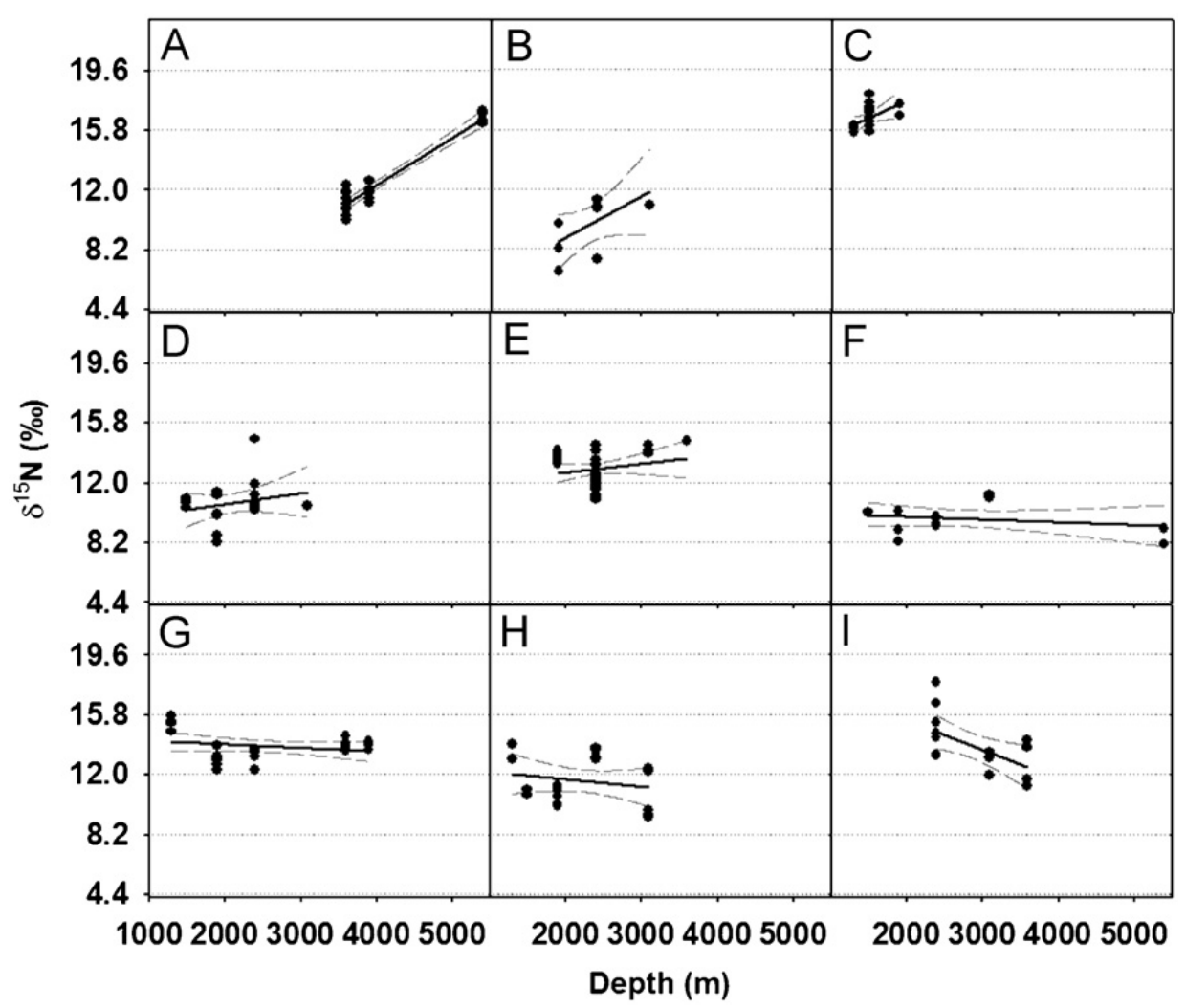

Fig. 5. Relationship between $\delta^{15} \mathrm{~N}$ signatures and water depth for (A) Bathyphellia margaritacea $\left(\delta^{15} \mathrm{~N}=0.25+0.0030\right.$ depth, $\left.r^{2}=0.94, p<0.001\right)$, (B) Bathycrinus cf. carpenteri $\left(\delta^{15} \mathrm{~N}=3.67+0.00263, r^{2}=0.33, p=0.11\right)$, (C) Bathybiaster vexillifer $\left(\delta^{15} \mathrm{~N}=13.28+0.00216, r^{2}=0.29, p=0.039\right),(\mathrm{D})$ Mesidothea megalura $\left(\delta^{15} \mathrm{~N}=9.17+0.00072, \quad r^{2}=0.05, \quad p=0.34\right), \quad(\mathrm{E})$ Lycodes frigidus $\left(\delta^{15} \mathrm{~N}=11.59+0.00052, \quad r^{2}=0.04, p=0.28\right), \quad(\mathrm{F})$ Cladorhiza gelida $\left(\delta^{15} \mathrm{~N}=10.23-0.00018, r^{2}=0.05, p=0.41\right),(\mathrm{G})$ Bythocaris spp. $\left(\delta^{15} \mathrm{~N}=14.35-0.00023\right.$ depth, $\left.r^{2}=0.06, p=0.24\right),(\mathrm{H})$ Mohnia mohni and Tacita danielsseni $\left(\delta^{15} \mathrm{~N}=12.63-0.00047\right.$ depth, $\left.r^{2}=0.04, p=0.42\right)$, and (I) Eurythenes gryllus $\left(\delta^{15} \mathrm{~N}=19.15-0.00186\right.$ depth, $\left.r^{2}=0.33, p=0.025\right)$. Dashed lines represent $95 \%$ confidence intervals.

sediments, particles from the nepheloid layer), which are used by a broader range of benthic biota. (2) The bottom POM value is more robust as it represents the mean $\delta^{15} \mathrm{~N}$ signature over a year. (3) It enabled a comparison with data from other studies in the Arctic, which use POM as a baseline (Hobson and Welch, 1992; Hobson et al., 1995; Iken et al., 2005; Søreide et al., 2006; Tamelander et al., 2006).

\subsubsection{Functional groups}

While we tried to assign the organisms to feeding groups as accurately as possible, it should be noted that $100 \%$ dependable autecological information is lacking for many deep-sea species. Furthermore, some species can switch between different feeding modes so as to capitalise on the most abundant source of food (e.g. Lopez and Levinton, 1987). Therefore, our results have to be treated with caution.

The majority of taxa sampled at HAUSGARTEN were suspension feeders, followed by predators/scavengers and deposit feeders. This may result from the use of an Agassiz trawl as the main sampling gear.

While it is not surprising that predators/scavengers had the highest $\delta^{15} \mathrm{~N}$ signatures at HAUSGARTEN it is puzzling that suspension feeders had higher $\delta^{15} \mathrm{~N}$ signatures than deposit feeders assuming that deposit feeders utilise organic matter that has been more often reworked
(Lopez and Levinton, 1987) and is therefore enriched in $\delta^{15} \mathrm{~N}$. Furthermore, suspension feeders may also feed on re-suspended matter (Iken et al., 2001) meaning that they utilise the same food repository as deposit feeders. However, several authors reported higher $\delta^{15} \mathrm{~N}$ values in tissues of suspension compared with deposit feeders (e.g. Iken et al., 2001; Lovvorn et al., 2005; Mintenbeck et al., 2007). Some of the taxa classified as suspension feeders may be micro- or macrophagous, e.g. sponges (Vacelet and Boury-Esnault, 1995) and anthozoans. Indeed, when these groups were omitted there were no significant differences between deposit and suspension feeders but the number tested also decreased from 124 to 36 individuals. Still, the vacuolated ectoderm of the anthozoan Bathyphellia margaritacea contained foreign particles, which indicates a true suspension-feeding mode (Riemann-Zürneck, 1997).

Predators/scavengers had the highest spread of $\delta^{15} \mathrm{~N}$ values, which encompassed 3.5 trophic levels. This is hardly surprising as the group included both scavengers and predators. It shows that they had a broad trophic niche width (Bearhop et al., 2004), possibly due to opportunistic dietary habits (Sweeting et al., 2005), which may indicate food limitation. More surprisingly, the $\delta^{15} \mathrm{~N}$ values of suspension feeders spanned three trophic levels. For comparison, in the German Bight, their range of $\delta^{15} \mathrm{~N}$ was only $1.5 \%$ (Dannheim, 2007). The broad range in $\delta^{15} \mathrm{~N}$ could be caused by size-selective feeding habits facilitated 
by the different feeding apparatus of different taxa. In addition, food limitation and temporary starvation may have contributed to the broad range observed as it can lead to an enrichment of $\delta^{15} \mathrm{~N}$ in animal tissues (Hobson et al., 1993; Frazer et al., 1997; Adams and Sterner, 2000; Kurle and Worthy, 2002; Olive et al., 2003; Cherel et al., 2005; Gaye-Siessegger et al., 2007). Nutritional stress may cause an increase of catabolic muscle protein breakdown. As the lighter nitrogen isotope is no longer replaced by dietary protein, the remaining tissues are enriched in the heavier isotope (increased fractionation).

The spread of deposit feeders over two trophic levels could be explained by different feeding strategies. While those feeding at a lower trophic level may select freshly deposited (isotopically lighter) surficial material, those occupying a higher level may feed less selectively on (sub-surface) material that has been repeatedly reworked and is therefore enriched in $\delta^{15} \mathrm{~N}$ (Iken et al., 2005). To avoid errors from misclassification, we did not distinguish between surface and sub-surface deposit feeders in our study.

\subsubsection{Species/taxon level}

The trophic positions revealed by our analyses did not always match our preconceptions. For example, the starfish Poraniomorpha tumida had the highest $\delta^{15} \mathrm{~N}$ signature recorded at HAUSGARTEN. The same was reported for a shallower food web in the Barents Sea (Tamelander et al., 2006), although at HAUSGARTEN the $\delta^{15} \mathrm{~N}$ of $P$. tumida was one trophic level higher. No information was found on the feeding behaviour, but the high $\delta^{15} \mathrm{~N}$ signatures strongly suggest a scavenging feeding mode. Similarly, Iken et al. (2001) reported highest $\delta^{15} \mathrm{~N}$ values for a starfish and a polychaete rather than for the known predatory/scavenging species of fish or amphipods of the Porcupine Abyssal Plain. The authors concluded that the latter were decoupled from the POMbased food web and fed on less frequently recycled abysso-pelagic prey and large food falls. Although food falls may occasionally subsidise fish populations, we found primarily small benthic invertebrates in fish stomachs from HAUSGARTEN (Bergmann, unpublished). Furthermore, the trophic level of fish was approximately one level higher than that of their identified prey. Therefore, the trophic level of $P$. tumida was probably higher because they fed at a higher trophic level than fish.

The enrichment between certain primary consumers and their food source (e.g. cumaceans, amphipods, sea pens) was much lower than the assumed fractionation of 3.8\% per trophic step. Congeneric cumaceans from the deep Arctic Canada Basin had also unexpectedly low $\delta^{15} \mathrm{~N}$ values, and Iken et al. (2005) suggested that they capitalise on very fresh and thus isotopically light phytodetritus. Post (2002b) stated that $3.4 \%$ is a valid estimate of trophic fractionation only when averaged over multiple trophic pathways and that any single trophic transfer is likely to range between $2 \%$ and $5 \%$ (Adams and Sterner, 2000). This may explain the low enrichment of some consumers relative to their food source.

\subsection{Bathymetric patterns in food supplies}

Only some $1-10 \%$ of the POM produced at the surface sinks to the sea floor (De La Rocha and Passow, 2007; Klages et al., 2003), and the amount of POM that reaches the seafloor decreases with increasing depth (Suess, 1980; Peinert et al., 2001) with the sharpest decrease in the twilight zone (Buesseler et al., 2007). Organisms from the deep Arctic seafloor are therefore subject to increasing food limitation. At HAUSGARTEN, the annual input of refractory particulate organic carbon and nitrogen beyond $300 \mathrm{~m}$ varies from 1.6 to 2.5 and 0.2 to $0.3 \mathrm{gm}^{-2}$, respectively (Bauerfeind et al., in revision), and food availability decreases as a function of depth (Soltwedel et al., 2005). Fig. 3 suggests that the quality of food supplies is also affected by depth. According to the 'temperature-substrate limitation hypothesis' (Mincks et al., 2005) the decrease in POM reaching the bottom, as well as decreasing temperatures along the HAUSGARTEN depth transect (Bauerfeind, unpublished data), may lead to decreasing microbially mediated mineralisation rates. Indeed, bacterial viability decreased as a function of depth at HAUSGARTEN (Quéric et al., 2004). The decreased turnover may have led in turn to the decreased $\delta^{15} \mathrm{~N}$ of sediments with depth. Ultimately, it is microbial breakdown that renders organic matter in sediments available to benthic biota (Lovvorn et al., 2005). Therefore, we consider depth as an indicator of food availability and suspect that the benthic assemblages and food webs present at different depths have been shaped by decreasing food supplies.

Mintenbeck et al. (2007) hypothesised that the $\delta^{15} \mathrm{~N}$ signatures of POM increase with increasing depth because of biodegradation in the water column of the Weddell Sea. However, the mean $\delta^{15} \mathrm{~N}$ values of POM samples from bottom and mid-water sediment traps recorded in our study were similar, which is corroborated by measurements from other years and HAUSGARTEN stations nearby (Bauerfeind, unpublished data). Similarly, Holmes et al. (1999) found that the $\delta^{15} \mathrm{~N}$ signatures of POM remain fairly constant below the euphotic zone. Accordingly, it could be argued that the $\delta^{15} \mathrm{~N}$ values of all organisms taken together were not affected by depth.

\subsection{Bathymetric patterns in functional groups}

Intriguingly, the $\delta^{15} \mathrm{~N}$ signatures of suspension feeders increased as a function of depth as also reported for the Weddell Sea (Mintenbeck et al., 2007). The comparison of bathymetric patterns in different functional groups in this study has to be treated with some caution as the species composition varied. However, a few species occurred at three or more depths allowing a direct comparison. As with all suspension feeders, the $\delta^{15} \mathrm{~N}$ signatures of $B$. margaritacea and the crinoid $B$. cf. carpenteri increased with increasing depth; the range of this increase exceeded one trophic level. This could have several explanations. (1) The HAUSGARTEN stations are positioned along the continental slope. Therefore, it is possible that benthic organisms also utilise particles that are transported 
down-slope or re-suspended sediments that may carry different $\delta^{15} \mathrm{~N}$ signatures compared with less degraded surface-POM. It could be hypothesised that the relative importance of such particles increases with depth since the quantity of surface POM decreases as a function of depth (Suess, 1980). Unfortunately, we were unable to sample the bottom POM at all of the HAUSGARTEN stations to verify this. (2) The bottom fauna may be increasingly exposed to food limitation and starvation. The increase in $\delta^{15} \mathrm{~N}$ with depth might thus reflect increasing nutritional stress due to lowered particle availability. However, the fact that suspension feeder densities at HAUSGARTEN increase as a function of depth (Jaeckisch, 2004) supports the first explanation.

The $\delta^{15} \mathrm{~N}$ of sponges were unexpectedly high for a group classified as suspension feeders. However, over recent years more and more sponges have been classified as carnivorous (Vacelet and Boury-Esnault, 1995; Vacelet, 2006; Ereskovsky and Willenz, 2007). Some of these (e.g. Cladorhiza, Esperiopsis) are closely related to those sampled in this study. Alternatively, high $\delta^{15} \mathrm{~N}$ values of sponges could be due to symbiotic bacteria (Iken et al., 2001). The mesohyl of Tentorium semisuberites from HAUSGARTEN harbours Archaea indicating "microbial farming" (Pape et al., 2006). Both carnivory and symbiotic bacteria may therefore explain the unexpectedly high $\delta^{15} \mathrm{~N}$ signatures recorded. Similar to those of predators/ scavengers, the $\delta^{15} \mathrm{~N}$ signatures of all sponges or of Cladorhiza gelida alone were not affected by depth. Macrophagy may be a more effective feeding strategy than microphagous suspension-feeding in food-limited deep-sea environments (Vacelet and Boury-Esnault, 1995) such as HAUSGARTEN.

Strikingly, the $\delta^{15} \mathrm{~N}$ signatures of deposit feeders followed the opposite trend: the $\delta^{15} \mathrm{~N}$ values decreased with increasing water depth. This is consistent with a reduction in bacterial viability and abundance of sediment-inhabiting bacteria with increasing water depth recorded at HAUSGARTEN (Quéric et al., 2004). As mentioned above, decreasing concentrations of organic matter and temperatures - as found along the HAUSGARTEN transect - may lead to decreased microbially mediated mineralisation rates (Mincks et al 2005). Organic matter in sediments is indigestible by macrofauna and becomes available to deposit feeders only through microbial breakdown (Lovvorn et al., 2005). Under oxic conditions, microbial degradation of organic matter leads to an enrichment of $\delta^{15} \mathrm{~N}$ in sediments (Macko and Estep, 1984; Holmes et al., 1999; Freudenthal et al., 2001). A decrease in bacterial abundance and viability with depth therefore also explains the recorded decrease in $\delta^{15} \mathrm{~N}$ of sediments with growing depth. The lower $\delta^{15} \mathrm{~N}$ signature of their food source is reflected, in turn, in the tissues of deposit feeders. Furthermore, as food supplies decrease with increasing depth, deposit feeders may rely increasingly on smaller particles, which have larger surface to volume ratios compared to larger particles. This may maximise food uptake (Lopez and Levinton, 1987). Small particles are characterised by lower $\delta^{15} \mathrm{~N}$ signatures (Rau et al., 1990). Their preferential ingestion may therefore also contribute to the decreasing $\delta^{15} \mathrm{~N}$ signatures recorded.

Since none of the deposit-feeding species was sampled at more than two depth strata we were unable to corroborate the depth-related patterns at the species level. Our findings concur with photographic evidence from HAUSGARTEN indicating decreasing surface deposit feeder densities as a function of depth (Jaeckisch, 2004).

The $\delta^{15} \mathrm{~N}$ values of predators/scavengers and of most individual predatory/scavenging taxa were not affected by depth. Likewise, Polunin et al. (2001) detected depthrelated differences only in two out of 20 species of predators/scavengers from the deep Mediterranean. Predators/scavengers may have to adopt a degree of omnivory to be able to make ends meet in environments as food-limited as the deep sea. Indeed, stomach content analysis of the same individual fish used for stable isotope analyses revealed that they fed on a mixture of benthic invertebrates (Bergmann, unpublished data: amphipods, cumaceans, copepods, isopods, tanaids, polychaetes) with different feeding modes. The opposite depth patterns in the $\delta^{15} \mathrm{~N}$ of suspension and deposit feeders may thus have been evened out in the tissues of their consumers.

By contrast, the $\delta^{15} \mathrm{~N}$ signatures of the amphipod Eurythenes gryllus decreased with depth. While this species has been reported from various baited trap and food fall experiments around the globe (e.g. Hessler et al., 1978; Premke et al., 2003) it may switch to different food sources, e.g. bottom POM, during periods of carrion scarcity (Charmasson, 1998). When removing the hindgut whilst sampling tissue from the abdomen, we also found sediments. If carrion becomes scarcer with depth E. gryllus may increasingly adopt a diet including particles or deposit-feeding prey. As argued above, these sources are increasingly depleted in $\delta^{15} \mathrm{~N}$, which may have caused the depth-related $\delta^{15} \mathrm{~N}$ depletion observed.

Surprisingly, the $\delta^{15} \mathrm{~N}$ signatures of the starfish $B$. vexillifer increased with increasing water depth. The dominant prey of conspecifics from the Rockall Trough included irregular echinoids, gastropods and bivalves (Tyler et al., 1993). As macrofaunal abundance and biomass decreases with increasing water depth in the HAUSGARTEN area (Włodarska-Kowalczuk et al., 2004), starfish may increasingly suffer starvation or feed on food falls reflected in growing $\delta^{15} \mathrm{~N}$ values.

\section{Conclusions}

The bathymetric patterns in $\delta^{15} \mathrm{~N}$ were different in all feeding groups. While the $\delta^{15} \mathrm{~N}$ signatures of predators/scavengers were not affected by depth, those of suspension feeders increased with depth, and the reverse was found for deposit feeders. Suspension feeders feed on POM, which decreases in availability with increasing depth. As a result, suspension feeders may rely increasingly on particles that trickle down the HAUSGARTEN slope and carry higher $\delta^{15} \mathrm{~N}$ values. The fact that suspension feeder densities increase with depth (Jaeckisch, 2004) indicates the importance of this food source at HAUSGARTEN. Deposit feeders feed on material recycled 
by sediment-inhabiting bacteria, which may be less and less reworked as their biomass decreases with depth (Quéric et al., 2004). The decreasing food availability may lead to the decreasing surface deposit feeder densities observed as a function of depth (Jaeckisch, 2004). Our results highlight the need for a depth-stratified approach to analyses of deep-sea food webs.

A more comprehensive sampling approach including samples from ice cores would allow us to determine the importance of sympagic input to deep benthic communities below the marginal ice zone. Extraction of lipids prior to stable isotope analyses, or the use of fatty acid trophic biomarkers, would enable us to infer the sources of carbon used by benthic organisms and increase the dietary resolution.

Our results cast new light on a food web located in an area that may already be affected by climate change or soon become so. Changes in food-web relationships could markedly alter benthic community and trophic structure, thereby influencing food availability up to apex consumers. Time-series measurements of the isotopic signature of longer-lived key benthic species may allow us to infer trophic changes in response to climate change.

\section{Acknowledgements}

We thank the officers and crews of RVs Polarstern and L'Atalante and the ROV team of "Victor 6000" for their support. S. Bury, A. Pappert and M. Volkenandt assisted with the preparation of samples. U. Struck undertook the isotope-ratio mass spectrometry analysis and K. Beyer gave practical instructions on the methodology. R. Bramber, A. Brandt, S. Gromisz, J. Guerrero, D. Jones, M. Kędra, P.R. Møller, U. Mühlenhardt-Siegel, D. Piepenburg, M. Ramos, A.V. Rogacheva, O.S. Tendal, M. Türkay, K. von Juterzenka, A. Warén and J.M Węsławski kindly identified fauna from reference samples and provided information on feeding habits. The suggestions of three anonymous referees improved an earlier version of the manuscript. This is publication awi-n17402 of the Alfred Wegener Institute for Polar and Marine Research.

\section{References}

Adams, T.S., Sterner, R.W., 2000. The effect of dietary nitrogen content on trophic level ${ }^{15} \mathrm{~N}$ enrichment. Journal Limnology and Oceanography 45, 601-607.

Adlandsvik, B., Gundersen, A.C., Nedreaas, K.H., Stene, A., Albert, O.T., 2004. Modelling the advection and diffusion of eggs and larvae of Greenland halibut (Reinhardtius hippoglossoides) in the north-east Arctic. Fisheries Oceanography 13, 403-415.

Bailey, D., King, N., Priede, I., 2007. Cameras and carcasses: historical and current methods for using artificial food falls to study deep-water animals. Marine Ecology Progress Series 350, 179-191.

Bauerfeind, E., Nöthig, E.-M., Beszczynska, A., Fahl, K., Kaleschke, L., Kreker, K., Klages, M., Soltwedel, T., Lorenzen, C., Wegner, J. Variations in vertical particle flux in the eastern Fram Strait $\left(79^{\circ} \mathrm{N} / 4^{\circ} \mathrm{E}\right)$ during 2000-2005. Results from the deep-sea long-term observatory HAUSGARTEN. Deep-Sea Research I, in revision.

Bearhop, S., Colin, E.A., Waldron, S., Fuller, R.A., MacLeod, H., 2004. Determining trophic niche width: a novel approach using stable isotope analysis. Journal of Animal Ecology 73, 1007-1012.
Bodungen, v.B., Wunsch, M., Fürderer, H., 1991. Sampling and analysis of suspended and sinking particles in the Northern North Atlantic. In: Hurd, D.C., Spencer, D.W. (Eds.), Marine Particles and Characterization. Geophysical Monograph 63, pp. 47-56.

Buesseler, K.O., Lamborg, C.H., Boyd, P.W., Lam, P.J., Trull, T.W., Bidigare, R.R., Bishop, J.K.B., Casciotti, K.L., Dehairs, F., Elskens, M., Honda, M. Karl, D.M., Siegel, D.A., Silver, M.W., Steinberg, D.K., Valdes, J., Van Mooy, B., Wilson, S., 2007. Revisiting carbon flux through the ocean's twilight zone. Science 316, 567-570.

Charmasson, S., 1998. Cycle du combustible nucléaire et milieu marin. Devenir des effluents rhodaniens en Méditerranée et des déchets immergés en Atlantique nord-est, Université Aix-Marseille II < http:// www-ist.cea.fr/publicea/exl-doc/00000037203.pdf $>$.

Cherel, Y., Hobson, K.A., Bailleul, F.R., Groscolas, R., 2005. Nutrition, physiology, and stable isotopes: new information from fasting and molting penguins. Ecology 86, 2881-2888.

Dannheim, J., 2007. Macrozoobenthic response to fishery-trophic interactions in highly dynamic coastal ecosystems. Ph.D. Thesis, University of Bremen, Bremerhaven.

Danovaro, R., Gambi, C., Dell'Anno, A., Corinaldesi, C., Fraschetti, S., Vanreusel, A., Vincx, M., Gooday, A.J., 2008. Exponential decline of deep-sea ecosystem functioning linked to benthic biodiversity loss. Current Biology 18, 1-8.

De La Rocha, C.L., Passow, U., 2007. Factors influencing the sinking of POC and the efficiency of the biological carbon pump. Deep-Sea Research Part II 54, 639-658.

Ereskovsky, A.V., Willenz, P., 2007. Esperiopsis koltuni sp. Nov. (Demospongiae: Poecilosclerida: Esperiopsidae), a carnivorous sponge from deep water of the Sea of Okhotsk (North Pacific). Journal of the Marine Biological Association of the UK 87 , 1379-1386.

Frazer, T., Ross, R., Quetin, L., Montoya, J., 1997. Turnover of carbon and nitrogen during growth of larval krill, Euphausia superba Dana: a stable isotope approach. Journal of Experimental Marine Biology and Ecology 212, 259-275.

Freudenthal, T., Wagner, T., Wenzhöfer, F., Zabel, M., Wefer, G., 2001. Early diagenesis of organic matter from sediments of the eastern subtropical Atlantic: evidence from stable nitrogen and carbon isotopes. Geochimica et Cosmochimica Acta 65, 1795-1808.

Gage, J., Tyler, P., 1991. Deep-Sea Biology: A Natural History of Organisms at the Deep-Sea Floor. Cambridge University Press, New York.

Gaye-Siessegger, J., Focken, U., Abel, H., Becker, K., 2007. Starvation and low feeding levels result in an enrichment of ${ }^{13} \mathrm{C}$ in lipids and ${ }^{15} \mathrm{~N}$ in protein of Nile tilapia Oreochromis niloticus L. Journal of Fish Biology 71, 90-100.

Grebmeier, J.M., Overland, J.E., Moore, S.E., Farley, E.V., Carmack, E.C., Cooper, L.W., Frey, K.E., Helle, J.H., McLaughlin, F.A., McNutt, S.L., 2006. A major ecosystem shift in the northern Bering Sea. Science 311, 1461-1464.

Hall, S., Raffaelli, D., 1993. Food webs: theory and reality. Advances in Ecological Research 24, 187-239.

Hessler, R.R., Ingram, C.L., Yayanos, A.A., Burnett, B.R., 1978. Scavenging amphipods from the floor of the Philippine Trench. Deep-Sea Research 25, 1029-1047.

Hobson, K.A., Wassenaar, L.I., 1999. Stable isotope ecology: an introduction. Oecologia 120, 312-313.

Hobson, K.A., Welch, H.E., 1992. Determination of trophic relationships within a high Arctic marine food web using $\delta^{13} \mathrm{C}$ and $\delta^{15} \mathrm{~N}$ analysis. Marine Ecology Progress Series 84, 9-18.

Hobson, K., Alisauskas, R., Clark, R., 1993. Stable-nitrogen isotope enrichment in avian tissues due to fasting and nutritional stress: implications for isotopic analyses of diet. The Condor 95, 388-394.

Hobson, K.A., Ambrose, W.G., Renaud, P.E., 1995. Sources of primary production, benthic-pelagic coupling, and trophic relationships within the Northeast Water Polynya: insights from $\delta^{13} \mathrm{C}$ and $\delta^{15} \mathrm{~N}$ analysis. Marine Ecology Progress Series 128, 1-10.

Holmes, M.E., Eichner, C., Struck, U., Wefer, G., 1999. Reconstruction of surface ocean nutrient utilization using stable nitrogen isotopes in sinking particles and sediments. In: Fischer, G., Wefer, G. (Eds.), The Use of Proxies in Paleoceanography: Examples from the South Atlantic. Springer, Berlin, pp. 447-468.

Hoste, E., Vanhove, S., Schewe, I., Soltwedel, T., Vanreusel, A., 2007. Spatial and temporal variations in deep-sea meiofauna assemblages in the marginal ice zone of the Arctic Ocean. Deep-Sea Research Part I $54,109-129$.

Iken, K., Brey, T., Wand, U., Voigt, J., Junghans, P., 2001. Food web structure of the benthic community at the Porcupine Abyssal Plain (NE Atlantic): a stable isotope analysis. Progress in Oceanography 50, 383-405. 
Iken, K., Bluhm, B.A., Gradinger, R., 2005. Food web structure in the high Arctic Canada Basin: evidence from $\delta^{13} \mathrm{C}$ and $\delta^{15} \mathrm{~N}$ analysis. Polar Biology 28, 238-249.

IPCC, 2007. Climate change 2007-The Physical Science Basis (Working Group I Contribution to the Fourth Assessment Report of the IPCC). Cambridge University Press, Cambridge.

Jacob, U., Mintenbeck, K., Brey, T., Knust, R., Beyer, K., 2005. Stable isotope food web studies: a case for standardized sample treatment. Marine Ecology Progress Series 287, 251-253.

Jaeckisch, N., 2004. Characterisation of the mega-epibenthic community along a depth gradient in the deep Fram Strait (Arctic Ocean). Dissertation, University of Hamburg.

Jones, E.G., Collins, M.A., Bagley, P.M., Addison, S., Priede, I.G., 1998. The fate of cetacean carcasses in the deep sea: observations on consumption rates and succession of scavenging species in the abyssal north-east Atlantic Ocean. Proceedings of the Royal Society London 265, 1119-1127.

Jumars, P.A., Wheatcroft, R.A., 1989. Responses of benthos to changing food quality and quantity, with a focus on deposit feeding and bioturbation. In: Berger, W.H., Smetacek, V.S., Wefer, G. (Eds.), Productivity of the Ocean: Present and Past. Wiley, Chichester, pp. 235-253.

Klages, M., Vopel, K., Bluhm, H., Brey, T., Soltwedel, T., Arntz, W.E., 2001. Deep-sea food falls: first observation of a natural event in the Arctic Ocean. Polar Biology 24, 292-295.

Klages, M., Boetius, A., Christensen, J.P., Deubel, H., Piepenburg, D., Schewe, I., Soltwedel, T., 2003. The benthos of Arctic Seas and its role for the carbon cycle at the seafloor. In: Stein, R., Macdonald, R.W. (Eds.). Springer, Heidelberg, pp. 139-167.

Kurle, C.M., Worthy, G.A.J., 2002. Stable nitrogen and carbon isotope ratios in multiple tissues of the northern fur seal Callorhinus ursinus: implications for dietary and migratory reconstructions. Marine Ecology Progress Series 236, 289-300.

Lopez, G.R., Levinton, J.S., 1987. Ecology of deposit-feeding animals in marine sediments. Quarterly Review of Biology 62 (3), 235-260.

Lovvorn, J.R., Cooper, L.W., Brooks, M.L., De Ruyck, C.C., Bump, J.K., Grebmeier, J.M., 2005. Organic matter pathways to zooplankton and benthos under pack ice in late winter and open water in late summer in the north-central Bering Sea. Marine Ecology Progress Series 291, 135-150.

Macko, S.A., Estep, M.L.F., 1984. Microbial alteration of stable nitrogen and carbon isotopic compositions of organic matter. Organic Geochemistry 6, 787-790.

McMahon, K., Ambrose, W.J., Johnson, B., Sun, M., Lopez, G., Clough, L., Carroll, M., 2006. Benthic community response to ice algae and phytoplankton in Ny, Ålesund Svalbard. Marine Ecology Progress Series 310, 1-14.

Mincks, S.L., Smith, C.R., De Master, D.J., 2005. Persistence of labile organic matter and microbial biomass in Antarctic shelf sediments: evidence of a sediment 'food bank'. Marine Ecology Progress Series 300, 3-19.

Mintenbeck, K., Jacob, U., Knust, R., Arntz, W.E., Brey, T., 2007. Depthdependence in stable isotope ratio $\delta^{15} \mathrm{~N}$ of benthic pom consumers: the role of particle dynamics and organism trophic guild. Deep-Sea Research Part I 54, 1015-1023.

Mintenbeck, K., Brey, T., Jacob, U., Knust, R., Struck, U., 2008. How to account for the lipid effect on carbon stable-isotope ratio $\left(\delta^{13} \mathrm{C}\right)$ : sample treatment effects and model bias. Journal of Fish Biology 72, 815-830.

Nürnberg, D., Wollenburg, I., Dethleff, D., Eicken, H., Kassens, H., Letzig, T., Reimnitz, E., Thiede, J., 1994. Sediments in Arctic sea ice: implications for entrainment, transport and release. Marine Geology 119, 185-214.

Olive, P.J.W., Pinnegar, J.K., Polunin, N.V.C., Richards, G., Welch, R., 2003. Isotope trophic-step fractionation: a dynamic equilibrium model. Journal of Animal Ecology 72, 608-617.

Pape, T., Hoffmann, F., Quéric, N.-V., Juterzenka, K.v., Reitner, J., Michaelis, W., 2006. Dense populations of Archaea associated with the demosponge Tentorium semisuberites Schmidt, 1870 from Arctic deep-waters. Polar Biology, 1432-2056.

Peinert, R., Bauerfeind, E., Gradinger, R., Haupt, O., Krumbholz, M., Peeken, I., Werner, I., Zeitzschel, B., 2001. Particle sources and vertical particle flux in the seasonally ice covered Greenland Sea. In: Schäfer, P., Ritzrau, W.R., Schlüter, M., Thiede, J. (Eds.), Northern North Atlantic: A Changing Environment. Springer, Heidelberg, pp. 69-79.

Polunin, N.V.C., Morales-Nin, B., Pawsey, W.E., Cartes, J.E., Pinnegar, J.K., Moranta, J., 2001. Feeding relationships in Mediterranean bathyal assemblages elucidated by carbon and nitrogen stable-isotope data. Marine Ecology Progress Series 220,13-23.

Post, D.M., 2002a. The long and short of food-chain length. Trends in Ecology and Evolution 17, 269-277.

Post, D.M., 2002b. Using stable isotopes to estimate trophic position: models, methods, and assumptions. Ecology Letters 83, 703-718.

Premke, K., Muyakshin, S., Klages, M., Wegner, J., 2003. Evidence for longrange chemoreceptive tracking of food odour in deep-sea scavengers by scanning sonar data. Journal of Experimental Marine Biology and Ecology 285-286, 283-294.

Quéric, N.-V., Soltwedel, T., Arntz, W.E., 2004. Application of a rapid direct viable count method to deep-sea sediment bacteria. Journal of Microbiological Methods 57, 351-367.

Rau, G.H., Teyssie, J.-L., Rassoulzadegan, F., Fowler, S.W., $1990 .{ }^{13} \mathrm{C} /{ }^{12} \mathrm{C}$ and ${ }^{15} \mathrm{~N} /{ }^{14} \mathrm{~N}$ variations among size-fractionated marine particles: implications for their origin and trophic relationships. Marine Ecology Progress Series 59, 33-38.

Riemann-Zürneck, K., 1997. The deep-sea anemones Bathyphellia margaritacea and Daontesia porcupina sp. nov. with comments on the family Bathyphelliidae. Journal of the Marine Biological Association UK 77, 361-374.

Sahling, H., Galkin, S.V., Salyuk, A., Greinert, J., Foerstel, H., Piepenburg, D., Suess, E., 2003. Depth-related structure and ecological significance of cold-seep communities-a case study from the Sea of Okhotsk. Deep-Sea Research Part I 50, 1391-1409.

Sakshaug, E., 2003. Primary and secondary production in the Arctic seas. In: Stein, R., Macdonald, R.W. (Eds.), The Organic Carbon Cycle in the Arctic Ocean. Springer, Heidelberg, pp. 57-81.

Schauer, U., Beszczynska-Möller, A., Walczowski, W., Fahrbach, E., Piechura, J., Hansen, E., 2008. Variation of measured heat flow through the Fram Strait between 1997 and 2006. In: Dickson, R.R., Meincke, J., Rhines, P. (Eds.), Arctic-Subarctic Ocean Fluxes: Defining the Role of the Northern Seas in Climate. Springer, Berlin, pp. 65-84.

Schlichtholz, P., Houssais, M.-N., 2002. An overview of the $\theta-S$ correlations in Fram Strait based on the MIZEX 84 data. Oceanologia $44,243-272$.

Sherwood, G.D., Rose, G.A., 2005. Stable isotope analysis of some representative fish and invertebrates of the Newfoundland and Labrador continental shelf food web. Estuarine, Coastal and Shelf Science 63, 537-549.

Smith, C.R., 1985. Food for the deep sea: Utilization, dispersal, and flux of nekton falls at the Santa Catalina Basin floor. Deep-Sea Research 32, 417-442.

Smith Jr., K.L., 1987. Food energy supply and demand: a discrepancy between particulate organic carbon flux and sediment community oxygen consumption in the deep ocean. Limnology and Oceanography 32, 201-220.

Smith, C.R., De Leo, F.C., Bernardino, A.F., Sweetman, A.K., MartinezArbizu, P.M., 2008. Abyssal food limitation, ecosystem structure and climate change. Trends in Ecology and Evolution 23, 518-528.

Soltwedel, T., Juterzenka, K.v., Premke, K., Klages, M., 2003. What a lucky shot! Photographic evidence for a medium-sized natural food-fall at the deep seafloor. Oceanologica Acta 26, 623-628.

Soltwedel, T., Bauerfeind, E., Bergmann, M., Budaeva, N., Hoste, E., Jaeckisch, N., Juterzenka, K.v., Matthiessen, J., Mokievsky, V., Nöthig, E.-M., Quéric, N.-V., Sablotny, B., Sauter, E., Schewe, I., Urban-Malinga, B., Wegner, J., Włodarska-Kowalczuk, M., Klages, M., 2005. HAUSGARTEN_-multidisciplinary investigations at a deepsea long-term observatory in the Arctic Ocean. Oceanography 18, 46-61.

Søreide, J.E., Hop, H., Carroll, M.L., Falk-Petersen, S., Hegseth, E.N., 2006. Seasonal food web structures and sympagic-pelagic coupling in the European Arctic revealed by stable isotopes and a two-source food web model. Progress in Oceanography 71, 59-87.

Suess, E., 1980. Particulate organic carbon flux in the oceans-surface productivity and oxygen utilization. Nature 288, 260-263.

Sweeting, C.J., Jennings, S., Polunin, N.V.C., 2005. Variance in isotopic signatures as a descriptor of tissue turnover and degree of omnivory. Functional Ecology 19, 777-784.

Tamelander, T., Renaud, P.E., Hop, H., Carroll, M.L., Ambrose, W.G.J., Hobson, K.A., 2006. Trophic relationships and pelagic-benthic coupling during summer in the Barents Sea Marginal Ice Zone, revealed by stable carbon and nitrogen isotope measurements. Marine Ecology Progress Series 310, 33-46.

Tyler, P.A., Gage, J.D., Paterson, G.J.L., Rice, A.L., 1993. Dietary constraints on reproductive periodicity in two sympatric deep-sea astropectinid seastars. Marine Biology 115, 267-277. 
Vacelet, J., 2006. New carnivorous sponges (Porifera, Poecilosclerida) collected from manned submersibles in the deep Pacific. Zoological Journal of the Linnean Society 148, 553-584.

Vacelet, J., Boury-Esnault, N., 1995. Carnivorous sponges. Nature 373, 333-335.

Wassmann, P., Bauerfeind, E., Fortier, W., Fukuchi, M., Hargrave, B., B, M., Noji, T., Nöthig, E.-M., Olli, K., Peinert, R., Sasaki, H., Shevchenko, V.,
2003. Particulate organic carbon flux to the Arctic Ocean sea floor. In: Stein, R., Macdonald, R.W. (Eds.), The Organic Carbon Cycle in the Arctic Ocean. Springer, Heidelberg, pp. 101-138.

Włodarska-Kowalczuk, M., Kendall, M.A., Węsławski, J.M., Klages, M. Soltwedel, T., 2004. Depth gradients of benthic standing stock and diversity on the continental margin at a high-latitude ice-free site (off Spitsbergen, $7^{\circ}$ N). Deep-Sea Research Part I 51, 1903-1914. 